\title{
Risk measure estimation under two component mixture models with trimmed data
}

DOI:

10.1080/02664763.2018.1517146

\section{Document Version}

Accepted author manuscript

Link to publication record in Manchester Research Explorer

\section{Citation for published version (APA):}

Abu Bakar, S. A., \& Nadarajah, S. (2019). Risk measure estimation under two component mixture models with trimmed data. Journal of Applied Statistics, 46(5), 835-852. https://doi.org/10.1080/02664763.2018.1517146

\section{Published in:}

Journal of Applied Statistics

\section{Citing this paper}

Please note that where the full-text provided on Manchester Research Explorer is the Author Accepted Manuscript or Proof version this may differ from the final Published version. If citing, it is advised that you check and use the publisher's definitive version.

\section{General rights}

Copyright and moral rights for the publications made accessible in the Research Explorer are retained by the authors and/or other copyright owners and it is a condition of accessing publications that users recognise and abide by the legal requirements associated with these rights.

\section{Takedown policy}

If you believe that this document breaches copyright please refer to the University of Manchester's Takedown Procedures [http://man.ac.uk/04Y6Bo] or contact uml.scholarlycommunications@manchester.ac.uk providing relevant details, so we can investigate your claim.

\section{OPEN ACCESS}




\title{
Risk measure estimation under two component mixture models with trimmed data
}

\author{
by \\ S. A. Abu Bakar \\ Institute of Mathematical Sciences, University of Malaya, Kuala Lumpur, Malaysia \\ saab@um.edu.my \\ S. Nadarajah \\ School of Mathematics, University of Manchester, United Kingdom \\ mbbsssn2@manchester.ac.uk
}

\begin{abstract}
Several two component mixture models from the transformed gamma and transformed beta families are developed to assess risk performance. Their common statistical properties are given and applications to real insurance loss data are shown. A new data trimming approach for parameter estimation is proposed using the maximum likelihood estimation method. Assessment with respect to Value-at-Risk and Conditional Tail Expectation risk measures are presented. Of all the models examined, the mixture of inverse transformed gamma-Burr distributions consistently provides good results in terms of goodness-of-fit and risk estimation in the context of the Danish fire loss data.
\end{abstract}

Keywords: Danish fire loss data; Heavy tailed distributions; Mixture models; Transformed gamma and transformed beta families

\section{Introduction}

A series of papers on loss data modeling have dealt with the long-familiar Danish fire insurance loss data. The history of this data can be traced back to 1990s when McNeil (1997) first used extreme value theory to describe its potential behavior. The idea was supported using alternative statistical techniques and graphical devices by Resnick (1997). Both authors, however, use only part of the data exceeding one million Danish Krone to understand the extreme constituent of the tail. The data has received much attention from the scientific community partly due to restricted access to other real loss data sets. In general, the Danish loss data share many common features with others of the same kind such as high skewness, a very small portion of extreme values, a high frequency of observations of a small scale and a low frequency of observations of a large scale.

The complete Danish loss data consist of 2492 observations resulting from fire insurance claims that were recorded for the period from 1980 to 1990 in millions of Danish Krone (DKK). The data were obtained from Copenhagen Reinsurance and were aligned to reflect inflation. Some statistical excerpts of the data are as follows: first and third quartiles are 1.1570 and 2.6450, respectively; mean and median are 3.0630 and 1.6340, respectively; lowest and highest observations are 0.3134 and 263.3000, respectively; standard deviation is 7.97670. These basic properties show a highly skewed representation in the tail of the observations. At present, the data can be obtained from various online sources and statistical software packages. One such source for the data is given later in this paper. 
A large number of papers applying the Danish loss data proposed parametric models best describing its characteristics in terms of goodness-of-fit. Lately, the focus has been directed towards a new statistical model made by piecing together two weighted distributions at a specified threshold known as a composite model. Cooray and Ananda (2005) pioneered the study in this stream by introducing a two-parameter composite lognormal-Pareto model with a restricted mixing weight and showed its performance compared to standard distributions such as lognormal, Pareto, inverse Gaussian, gamma and Weibull distributions. Scollnik (2007) addressed the restricted issue by proposing a flexible mixing weight and found better results for handling the data. Various other composite models have been introduced thereafter and applied to the Danish loss data: Pigeon and Denuit (2011) proposed a composite model with a random threshold; Scollnik and Sun (2012) suggested several variations of the Weibull-Pareto composite model; some criteria for composite models based on the lognormal distribution were proposed by Nadarajah and Bakar (2014); Bakar et al. (2015) introduced a general procedure of constructing composite models for any two underlying distributions by expressing the threshold and the mixing weight in terms of other parameters of the model; Calderín-Ojeda (2015) proposed a new procedure to develop composite models based on a mode matching principle at the threshold.

Apart from the composite models, attempts to describe the Danish loss data were also made with other classes of distributions. For example, a class of log phase-type distributions was introduced by Ahn et al. (2012); Eling (2012) fitted skew-normal and skew-Student distributions considering a transformation kernel approach for benchmarking; Asgharzadeh et al. (2016) proposed a generalized inverse Lindley distribution and compared its fit to common heavy tailed distributions.

Several authors have used estimation methods based on trimming to model the Danish loss data: Brazauskas and Kleefeld (2009) used a method based on trimmed moments to estimate models for the Danish loss data; Ruckdeschel and Horbenko (2013) used a trimmed likelihood method to estimate models for the Danish loss data.

Nevertheless, not much has been realized on the multimodality of the Danish data. All the studies mentioned so far employ models that feature unimodality. More recently, Miljkovic and Grün (2016) initiated the use of non-Gaussian mixture models to describe this feature on the Danish loss data and showed their significance in risk estimation. The authors proposed the use of the Expected-Maximization algorithm using three initialization strategies for parameter estimation. Although several other studies had considered mixture models in loss modeling, all these authors used either dissimilar assessment approaches than those commonly used or different sets of loss data, some of which are not made available due to confidentiality issues, see for instance Lee and Lin (2010) and Bernardi et al. (2012).

All of the aforementioned authors showed practical importance of their proposed models in describing the tail behavior using quantiles or some well-established risk measures. Practitioners need to weigh many factors before any view can be made on financial positions of companies. Wrong decisions can lead to a company being insolvent. One factor for consideration is the risk measure. Value-at-Risk (VaR) and Conditional Tail Expectation (CTE) are among the popular risk measures applied in the insurance industry. Normally, theoretical measures are compared to empirical estimates in order to assess the appropriateness of fitted models.

Seeing the potential of mixture models in risk estimation, two component mixture models are proposed in this paper. Components of these models are based on the transformed beta and transformed gamma families. In Section 2, statistical properties of these models are given. In addition, a data trimming approach for parameter estimation, assessment methods for the proposed models grounded on information criteria and subsequently two important risk measures are discussed in 
this section. Section 3 applies the mixture models to the Danish loss data and assesses their performances in term of goodness-of-fit. Section 3 also compares theoretical and empirical estimates of risk measures. The paper is concluded in Section 4.

\section{Methodology}

In this section, we propose several two component mixture models and provide their basic statistical properties. Two component mixture models have shown sufficient reliability in terms of goodnessof-fit to loss data, see Miljkovic and Grün (2016). In general, the mixture model has the following general probability density function

$$
f(x)=w_{1} f_{1}(x)+w_{2} f_{2}(x), \quad x>0,
$$

where $w_{i}, i=1,2$ are the weights and $f_{i}(x), i=1,2$ are the underlying probability density functions representing two components of the mixture model having equal support on the real line. In the case of the loss data studied in this paper, $x$ is restricted to $x>0$. It is necessary that the total weights add up to one in order for (1) to serve as a valid probability density function. Throughout the paper, the weights are expressed in alternate forms with $w_{1}=\frac{1}{1+\phi}$ and $w_{1}=\frac{\phi}{1+\phi}$, where $\phi>0$.

Thus, the two component mixture model with probability density function in (1) has the following cumulative distribution function

$$
F(x)=\frac{1}{1+\phi} F_{1}(x)+\frac{\phi}{1+\phi} F_{2}(x), \quad x>0 .
$$

The non-central moment of order $r$ is

$$
E\left[X^{r}\right]=\frac{1}{1+\phi} E_{1}\left(X^{r}\right)+\frac{\phi}{1+\phi} E_{2}\left(X^{r}\right) .
$$

The moment generating function is

$$
M_{X}(t)=\frac{1}{1+\phi} M_{1}(t)+\frac{\phi}{1+\phi} M_{2}(t)
$$

provided that $M_{1}(t)$ and $M_{2}(t)$ exist. Many properties of the two component mixture model can be derived from the corresponding properties of the underlying component distributions. Thus closed form expressions for properties of the underlying components will ensure closed form expressions for the same properties of the mixture model.

It is important to mention that the models discussed here are based on mixture of transformed beta and transformed gamma families. These families exhibit high skewness in the tails of the distributions and therefore represent suitable candidates for modeling loss data. They are well documented in Klugman et al. (2012). Some of their particular cases are discussed in Venter (1983) and Klugman and Parsa (1999).

The transformed gamma distribution has the following probability density function

$$
g(x)=\frac{\tau u^{\kappa} \exp (-u)}{x \Gamma(\kappa)}, \quad x>0
$$

where $\kappa, \tau$ and $\theta$ are parameters greater than zero and $u=(x / \theta)^{\tau}$. It reduces to the: 
- inverse transformed gamma distribution when $u=(x / \theta)^{-\tau}$;

- gamma distribution when $u=(x / \theta)^{\tau}$ and $\tau=1$;

- inverse gamma distribution when $u=(x / \theta)^{-\tau}$ and $\tau=1$;

- Weibull distribution when $u=(x / \theta)^{\tau}$ and $\kappa=1$;

- inverse Weibull distribution when $u=(x / \theta)^{-\tau}$ and $\kappa=1$;

- exponential distribution when $u=(x / \theta)^{\tau}$ and $\kappa=\tau=1$;

- inverse exponential distribution when $u=(x / \theta)^{-\tau}$ and $\kappa=\tau=1$.

The transformed beta distribution has the following probability density function:

$$
h(x)=\frac{\Gamma(\alpha+\gamma)}{\Gamma(\alpha) \Gamma(\gamma)} \frac{\beta(x / \lambda)^{\beta \gamma}}{x\left[1+(x / \lambda)^{\beta}\right]^{\alpha+\gamma}}, \quad x>0,
$$

where $\alpha, \beta, \lambda$ and $\gamma$ are parameters greater than zero. It reduces to the:

- Burr distribution when $\gamma=1$;

- inverse Burr distribution when $\alpha=1$;

- generalized Pareto distribution when $\beta=1$;

- Pareto distribution when $\beta=\gamma=1$;

- inverse Pareto distribution when $\alpha=\beta=1$;

- paralogistic distribution when $\alpha=\beta$ and $\gamma=1$;

- inverse paralogistic distribution when $\beta=\gamma$ and $\alpha=1$;

- $\log \operatorname{logistic}$ distribution when $\alpha=\gamma=1$.

Our designation of the two mixture models considers the inverse transformed gamma distribution for $f_{1}(x)$ and one of the various distributions from the transformed beta and transformed gamma families for $f_{2}(x)$. As such, when $f_{2}(x)$ belongs to the transformed beta family, nine models are considered: the inverse transformed gamma-transformed beta (ITG-TB), the inverse transformed gamma-Burr (ITG-B), the inverse transformed gamma-inverse Burr (ITG-IB), the inverse transformed gamma-generalized Pareto (ITG-GP), the inverse transformed gamma-Pareto (ITG-P), the inverse transformed gamma-inverse Pareto (ITG-IP), the inverse transformed gammaparalogistic (ITG-PL), the inverse transformed gamma-inverse paralogistic (ITG-IPL) and the inverse transformed gamma-loglogistic (ITG-LL) models.

When $f_{2}(x)$ belongs to the transformed gamma family, eight models are considered: the inverse transformed gamma-transformed gamma (ITG-TG), the inverse transformed gamma-inverse transformed gamma (ITG-ITG), the inverse transformed gamma-gamma (ITG-G), the inverse transformed gamma-inverse gamma (ITG-IG), the inverse transformed gamma-Weibull (ITG-W), the inverse transformed gamma-inverse Weibull (ITG-IW), the inverse transformed gamma-exponential (ITG-E) and the inverse transformed gamma-inverse exponential (ITG-IE) models. 
Figure 1 shows the density functions of several mixture models for selected parameter values. We observe that each model can accommodate unimodal and multimodal density functions.

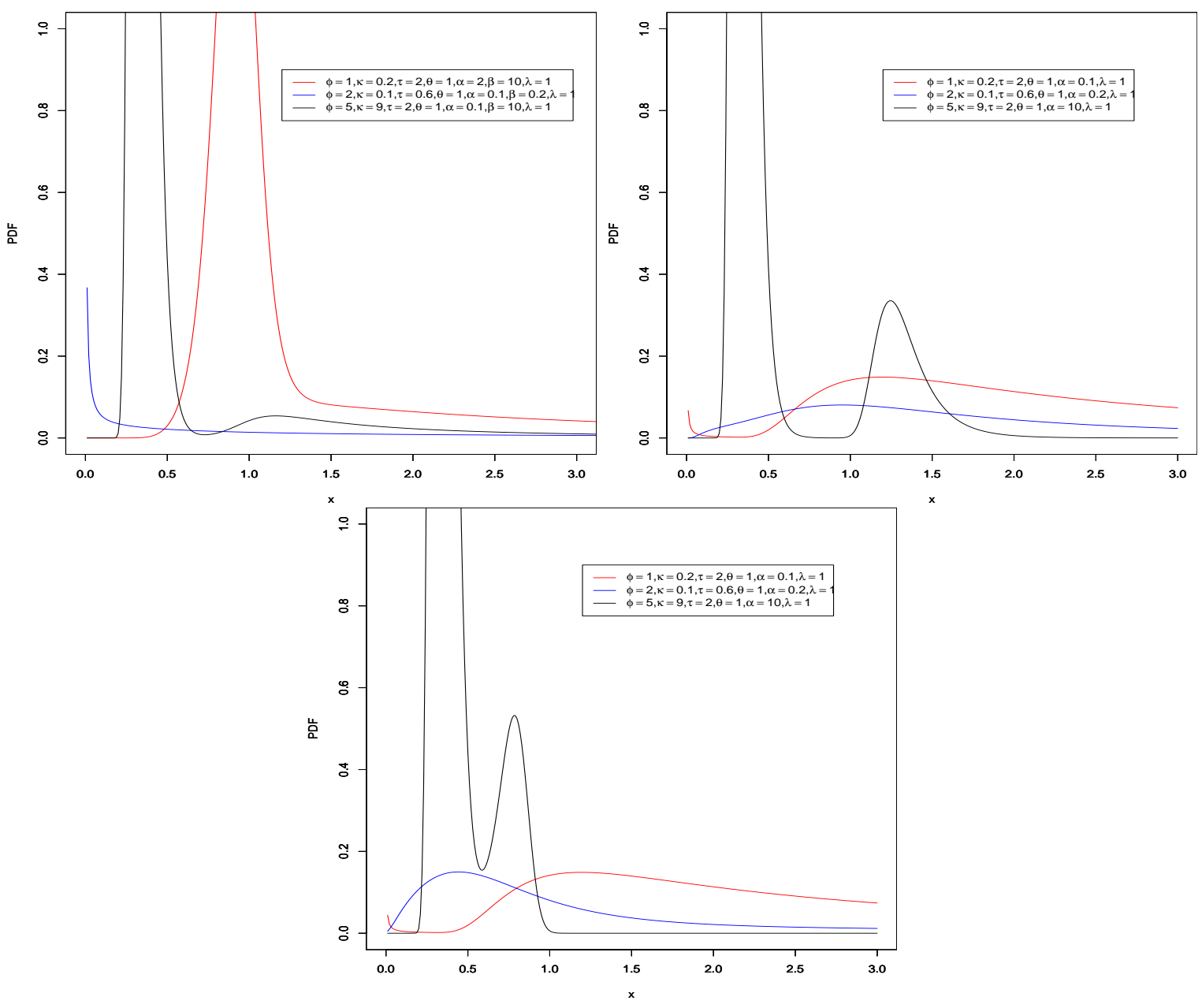

Figure 1: Probability density functions of the ITG-B (top left), ITG-IPL (top right) and ITG-PL (bottom) models for selected parameter values.

\subsection{Parameter estimation and model assessment}

Parameter estimation of the mixture models is performed using the maximum likelihood method. Prior to this, data trimming is proposed for unusual traits. These unusual traits are classified as data with logarithmic ratio based on maximum value being larger than minus 1 . This portion is considered to represent a substantial deviation of attributes and therefore excluded for the purpose of parameter estimation. The excluded data also referred to as extreme values for loss data can be easily observed by sorting the data or via a graphical plot. The idea is based on the properties of the natural logarithm, enabling the process of capturing extreme losses.

Given a set of data with $n$ observations, $\boldsymbol{\Psi}=\left\{x_{1}, x_{2}, x_{3}, \ldots, x_{n}\right\}$, let $\boldsymbol{\psi}=\left\{\psi_{1}, \psi_{2}, \ldots, \psi_{m}\right\}$ be a set of trimmed data such that $\boldsymbol{\psi} \subset \boldsymbol{\Psi}$. Then $x_{i} \in \boldsymbol{\psi}$ when it does not satisfy the following 
condition

$$
\ln \left[\frac{x_{i}}{\max (\Psi)}\right]>-1
$$

for $i=1,2,3, \ldots, n$. Note that $m \leq n$. Throughout the study, the trimmed data refers to the original data after excluding observations satisfying (2).

The log-likelihood function for the purpose of parameter estimation is given by

$$
L(\boldsymbol{\theta} \mid \boldsymbol{\psi})=\sum_{i=1}^{m}\left\{\ln \left[f_{1}\left(\psi_{i}\right)+\phi f_{2}\left(\psi_{i}\right)\right]\right\}-m \ln (1+\phi),
$$

where $\boldsymbol{\theta}=\{\phi, \ldots\}$ is the set of parameters of the mixture model.

Next, estimated parameters obtained from the optimization of (3) are substituted into the loglikelihood function with complete data. The log-likelihood function for the complete data is given by

$$
L(\widehat{\boldsymbol{\theta}} \mid \boldsymbol{\Psi})=\sum_{i=1}^{n}\left\{\ln \left[f_{1}\left(x_{i}\right)+\phi f_{2}\left(x_{i}\right)\right]\right\}-n \ln (1+\phi)
$$

where $\widehat{\boldsymbol{\theta}}$ denotes the estimated parameters. Equation (4) is intended for model assessment and selection as will be outlined below.

Three selection criteria are employed to assess the model performance, namely, the Negative Log-Likelihood (NLL), Akaike Information Criterion (AIC) and the Schwarz Bayesian Criterion (SBC). The last one is also known as the Bayesian Information Criterion. The formulas for the criteria used are as follows:

- $N L L=-L(\widehat{\boldsymbol{\theta}} \mid \boldsymbol{\Psi})$,

- $A I C=2 k+2 \cdot N L L$,

- $S B C=k \ln (n)+2 \cdot N L L$,

where $k$ and $n$ are the number of estimated parameters and number of observations, respectively. The lower the values of these criteria the better the fit to the data.

\section{$2.2 \quad$ Risk measures}

Two commonly used risk measures are discussed in order to assess the suitability of the fitted theoretical mixture models to the empirical estimates. Let $X$ denote a loss random variable, then the theoretical estimate of the $\mathrm{VaR}$ at $\alpha$ probability level is

$$
\operatorname{VaR}(\alpha)=F_{X}^{-1}(\alpha)
$$

where $F_{X}^{-1}(\cdot)$ denotes the inverse cumulative distribution function of $X$. The empirical estimate is found as the quantile value at probability level $\alpha$ :

$$
\operatorname{VaR}(\alpha)=\widehat{F}_{X}^{-1}(\alpha)
$$


where $\widehat{F}_{X}^{-1}(\cdot)$ denotes the empirical inverse cumulative distribution function.

The second risk measure considered here is the CTE. CTE considers the expected value of loss beyond the VaR at probability level $\alpha$. The theoretical CTE is

$$
C T E(\alpha)=\frac{1}{1-\alpha} \int_{\alpha}^{1} F_{X}^{-1}(s) d s
$$

Its empirical estimate is obtained as the average sum of all observations greater than the estimated VaR:

$$
C T E(\alpha)=\frac{1}{w} \sum_{i=1}^{w} r_{i}
$$

where $r_{i}$ for $i=1,2, \ldots, w$ are the empirical data greater than $\widehat{F}_{X}^{-1}(\alpha)$.

\section{Results and discussion}

In this section, results for seventeen mixture models based on the transformed beta and transformed gamma families as described earlier are provided with the aim of assessing risks. These models were fitted to trimmed Danish fire insurance data. Discussions on the Danish data are provided in numerous studies and are mentioned in Section 1 of this paper. In brief, this data consist of 2492 observations related to fire loss claim for the period between 1980 to 1990. The data were recorded in millions of Danish Krone with an aligned inflation factor. The empirical variance equals to 63.63 and three observations (with the values of 144.6576, 152.4132 and 263.2504) are found to have higher logarithmic ratio than minus one. Estimation of parameters for the mixture models was made by excluding these figures.

Table 1 summarizes the estimation results for mixture models with the second component belonging to the transformed beta family. Three selection criteria were employed, that is, the NLL, the AIC and the SBC. The lower the values of these criteria the better the fit of the mixture model to the Danish data. Comparison of models with equal number of parameters can be done using NLL. On the other hand, AIC and SBC penalize the number of parameters incorporated in a model and thus are suitable for comparison across models with varying complexity. It is important to mention that the NLL values reported here are found by substituting the estimated parameters obtained by maximizing the log-likelihood function with trimmed data into the log-likelihood function for complete data. However, for comparison purposes, values for the complete data are also provided (in brackets).

From Table 1, we observe that the ITG-B model gives the lowest AIC value followed by the ITGPL model and then the ITG-IPL model. The ITG-PL model gives the lowest SBC value followed by the ITG-B model and then the ITG-IPL model. The differences in these models with others proposed are not huge. These three models also perform better than the two component mixture of Burr models at least in terms of the SBC values. The two component mixture of Burr models was the best fitted model reported by Miljkovic and Grün (2016) (values of 3786.900, 7587.800 and 7628.546 were reported for NLL, AIC and SBC, respectively). Note that the three models above consist of seven, six and six parameters, respectively. Various performances are shown by the mixture models in Table 1 in comparison to mixtures of Burr models. All the mixture models excluding the ITG-IB, ITG-P and ITG-LL models show better values for the AIC and SBC than 
the best composite Weibull-Burr model proposed by Calderín-Ojeda (2015) with reported values of 3818.82, 7645.64 and 7668.92 for the NLL, AIC and SBC, respectively.

Table 1: Estimated values of NLL, AIC and SBC for mixture models with second component belonging to the transformed beta family (values in brackets correspond to the complete data set).

\begin{tabular}{|c|c|c|c|c|}
\hline Mixture models & $\begin{array}{c}\text { Number of } \\
\text { estimated parameters }\end{array}$ & NLL & $\mathrm{AIC}$ & $\mathrm{SBC}$ \\
\hline \multirow[t]{2}{*}{ ITG-TB } & 8 & $(3802.128)$ & $(7620.256)$ & $(7666.823)$ \\
\hline & & 3793.856 & 7603.712 & 7650.279 \\
\hline \multirow[t]{2}{*}{ ITG-B } & 7 & $(3788.123)$ & $(7590.246)$ & $(7630.991)$ \\
\hline & & 3785.672 & 7585.344 & 7626.090 \\
\hline \multirow[t]{2}{*}{ ITG-IB } & 7 & $(3924.832)$ & $(7863.664)$ & $(7904.409)$ \\
\hline & & 3932.253 & 7878.507 & 7919.252 \\
\hline \multirow[t]{2}{*}{ ITG-GP } & 7 & $(3793.655)$ & $(7601.310)$ & $(7642.056)$ \\
\hline & & 3795.445 & 7604.891 & 7645.637 \\
\hline \multirow[t]{2}{*}{ ITG-P } & 6 & $(3846.816)$ & $(7705.632)$ & $(7740.557)$ \\
\hline & & 3845.978 & 7703.956 & 7738.881 \\
\hline \multirow[t]{2}{*}{ ITG-IP } & 6 & $(3800.299)$ & $(7612.598)$ & $(7647.523)$ \\
\hline & & 3800.884 & 7613.768 & 7648.693 \\
\hline \multirow[t]{2}{*}{ ITG-PL } & 6 & $(3788.902)$ & $(7589.804)$ & $(7624.729)$ \\
\hline & & 3788.873 & 7589.746 & 7624.671 \\
\hline \multirow[t]{2}{*}{ ITG-IPL } & 6 & $(3789.921)$ & $(7591.843)$ & $(7626.768)$ \\
\hline & & 3789.672 & 7591.343 & 7626.268 \\
\hline \multirow[t]{2}{*}{ ITG-LL } & 6 & $(3854.597)$ & $(7721.195)$ & $(7756.120)$ \\
\hline & & 3854.665 & 7721.330 & 7756.255 \\
\hline
\end{tabular}

Models presented in Table 2 assume the transformed gamma family as the second component of the mixture models. None of the models in Table 2 resulted in a better fit than the ITG-B, ITG-IPL, ITG-PL models. The best fitted model within this family is the ITG-IG model with respect to the AIC and the ITG-E model with respect to the SBC. Similar to the previous result, all models in Table 2 excluding the ITG-TG, ITG-G, ITG-W and ITG-IW models perform better than the composite Weibull-Burr model. 
Table 2: Estimated values of NLL, AIC and SBC for mixture models with second component belonging to the transformed gamma family (values in brackets correspond to the complete data set).

\begin{tabular}{lcccc} 
Mixture models & $\begin{array}{c}\text { Number of } \\
\text { estimated parameters }\end{array}$ & NLL & AIC & SBC \\
ITG-TG & 7 & $(3825.021)$ & $(7666.042)$ & $(7712.609)$ \\
& & 3821.532 & 7659.064 & 7705.631 \\
ITG-ITG & 7 & $(3797.648)$ & $(7609.297)$ & $(7650.043)$ \\
& & 3805.058 & 7624.115 & 7664.861 \\
ITG-G & 6 & $(3802.169)$ & $(7616.339)$ & $(7651.264)$ \\
& & 3860.177 & 7732.353 & 7767.278 \\
ITG-IG & 6 & $(3799.366)$ & $(7610.732)$ & $(7645.657)$ \\
& \multirow{2}{*}{6} & 3799.518 & 7611.035 & 7645.960 \\
ITG-W & 6 & $(3972.072)$ & $(7956.144)$ & $(7991.069)$ \\
& \multirow{2}{*}{6} & 3821.375 & 7654.750 & 7689.675 \\
ITG-IW & & $(3819.280)$ & $(7650.560)$ & $(7685.485)$ \\
& 5 & 3820.417 & 7652.835 & 7687.760 \\
ITG-E & & $(3801.137)$ & $(7612.274)$ & $(7641.379)$ \\
& \multirow{2}{*}{5} & 3801.088 & 7612.175 & 7641.280 \\
ITG-IE & 5 & $(3809.623)$ & $(7629.245)$ & $(7658.349)$ \\
& & 3805.569 & 7621.138 & 7650.243
\end{tabular}

Estimated parameters and standard errors for the three best fitted mixture models are presented in Table 3. Note that all the parameters take values greater than zero including $\phi$ due to the design proposed. Graphical plots of the densities for the three mixture models are shown in Figure 2. 
Table 3: Estimated parameters, their standard errors and simulated 95 percent confidence intervals for the ITG-B, ITG-IPL and ITG-PL models.

\begin{tabular}{|c|c|c|c|c|}
\hline \multirow{8}{*}{$\begin{array}{l}\text { Mixture models } \\
\text { ITG-B }\end{array}$} & \multicolumn{3}{|c|}{ Estimated parameters with CI } & \multirow{2}{*}{$\begin{array}{c}\text { Standard errors with CI } \\
0.109(0.0501,1.001)\end{array}$} \\
\hline & $\widehat{\phi}$ & $=$ & $0.320(0.328,21.848)$ & \\
\hline & $\widehat{\kappa}$ & $=$ & $0.072(0.057,5.492)$ & $0.015(0.001,0.425)$ \\
\hline & $\widehat{\tau}$ & $=$ & $18.967(6.032,29.972)$ & $3.356(0.134,1.569)$ \\
\hline & $\widehat{\theta}$ & $=$ & $0.858(0.847,1.239)$ & $0.007(0.004,0.007)$ \\
\hline & $\widehat{\alpha}$ & $=$ & $0.318(0.078,0.401)$ & $0.085(0.002,0.033)$ \\
\hline & $\widehat{\beta}$ & $=$ & $5.311(5.532,16.687)$ & $0.696(0.159,0.462)$ \\
\hline & $\widehat{\gamma}$ & $=$ & $1.400(0.962,1.454)$ & $0.100(0.008,0.046)$ \\
\hline \multirow[t]{6}{*}{ ITG-IPL } & $\widehat{\phi}$ & $=$ & $0.319(0.000,1.092)$ & $0.065(0.000,0.058)$ \\
\hline & $\widehat{\kappa}$ & $=$ & $0.043(0.107,0.663)$ & $0.010(0.002,0.014)$ \\
\hline & $\widehat{\tau}$ & $=$ & $28.189(2.522,12.213)$ & $6.084(0.043,230)$ \\
\hline & $\widehat{\theta}$ & $=$ & $0.847(0.875,1.542)$ & $0.006(0.004,0.042)$ \\
\hline & $\widehat{\alpha}$ & $=$ & $2.938(0.282,29.029)$ & $0.177(0.82, \infty)$ \\
\hline & $\widehat{\lambda}$ & $=$ & $1.172(0.000,3.705)$ & $0.057(0.000,0.618)$ \\
\hline \multirow[t]{6}{*}{ ITG-PL } & $\widehat{\phi}$ & $=$ & $0.135(0.003,0.144)$ & $0.027(0.002,0.026)$ \\
\hline & $\widehat{\kappa}$ & $=$ & $0.065(0.003,0.144)$ & $0.011(0.002,0.026)$ \\
\hline & $\widehat{\tau}$ & $=$ & $18.734(11.913,25.976)$ & $2.996(0.233,0.516)$ \\
\hline & $\widehat{\theta}$ & $=$ & $0.858(0.852,0.885)$ & $0.006(0.003,0.005)$ \\
\hline & $\widehat{\alpha}$ & $=$ & $4.799(0.734,4.419)$ & $0.477(0.094,0.846)$ \\
\hline & $\widehat{\lambda}$ & $=$ & $2.430(1.063,9.850)$ & $0.113(0.093,5.563)$ \\
\hline
\end{tabular}

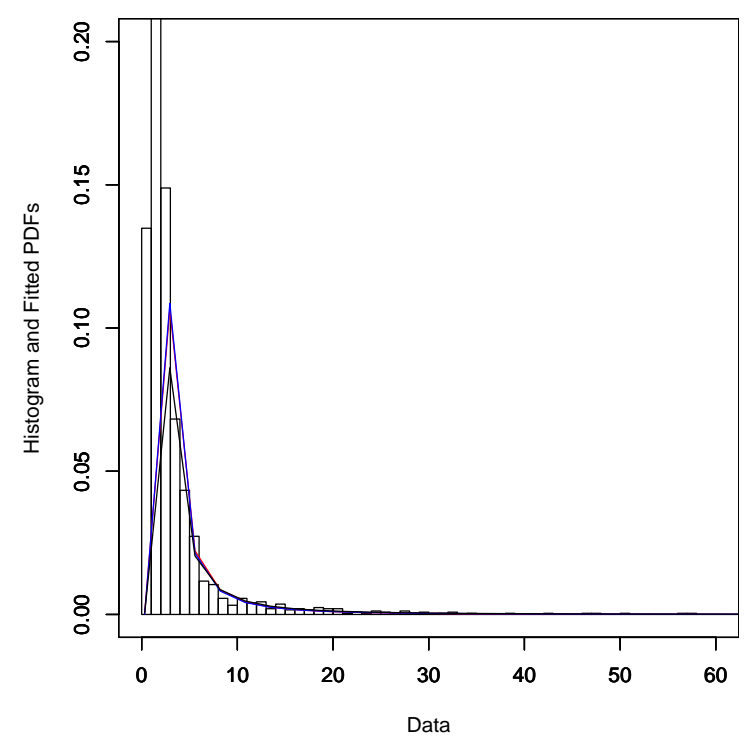

Figure 2: Fitted densities of the ITG-B (red), ITG-IPL (blue) and ITG-PL (black) models with estimated parameters computed using the data trimming approach.

In addition, comparison between the three best fitted models using Kolmogorov-Smirnov and 
Anderson-Darling tests are presented in Table 4. Both the ITG-B and ITG-IPL models provide adequate fits at the five percent level of significance. The ITG-B model provides the better fit of the two with respect to both tests. The ITG-PL model does not provide an adequate fit at the five percent level of significance.

Table 4: Tests statistics and $p$-values for the three best fitted models.

$\begin{array}{lcccc}\text { Model } & \text { KS statistic } & p \text {-value } & \text { AD statistic } & p \text {-value } \\ \text { ITG-B } & 0.020 & 0.253 & 0.852 & 0.445 \\ \text { ITG-IPL } & 0.023 & 0.158 & 1.116 & 0.302 \\ \text { ITG-PL } & 0.029 & 0.034 & 2.852 & 0.033\end{array}$

Further analysis to validate the three models can be performed using the likelihood ratio test. Under the null hypothesis that there is no improvement of the alternative model over the null model, the test statistic is:

$$
D=2\left[N L L_{0}-N L L_{1}\right]
$$

where $N L L_{0}$ and $N L L_{1}$ denote the negative log-likelihood values for the null and alternative models, respectively. $D$ is assumed to follow a chi-square distribution with degree of freedom equal to the difference in the number of parameters between the two compared models.

Considering the ITG-B and ITG-TB models as the null and alternative models, the test statistic is $D=2(7603.712-7585.344)=36.736$. With $D=36.736$ and one degree of freedom, the $p$-value is $1.353 \times 10^{-9}$. So, there is strong evidence to reject the null hypothesis and that the ITG-TB model is a significant improvement over the ITG-B model. Next, comparing the ITGIPL and ITG-TB models, the test statistic equals $D=2(7603.712-7589.746)=27.932$. With $D=27.932$ and two degrees of freedom, the $p$-value is $8.603 \times 10^{-7}$. Again, there is strong evidence to reject the null hypothesis and that the ITG-TB model is a significant improvement over the ITG-IPL model. Finally, comparing the ITG-PL and ITG-TB models, the test statistic equals $D=2(7603.712-7591.343)=24.738$. With $D=24.738$ and two degrees of freedom, the $p$-value is $4.248 \times 10^{-6}$. Yet again, there is strong evidence to reject the null hypothesis and that the ITG-TB model is a significant improvement over the ITG-PL model.

\subsection{Results on risk estimation}

In what follows, estimates of VaR and CTE are provided. The empirical estimates of these risk measures computed based on the complete Danish loss data are presented in the first row of Table 5 while the remaining rows represent the theoretical estimate found by (5) and (6) for VaR and CTE, respectively. Only the three best fitting mixture models found earlier are examined for their adherence to the empirical estimate. Note that both risk measures are computed at the 99 percent probability level and thus they may differ to a great extent from the empirical estimate depending on the tail of theoretical distributions.

Among the three models, the estimate of VaR by the ITG-B model shows the closest adherence to the empirical one. This is lower than the VaR estimate of the mixture of Burr models proposed by Miljkovic and Grün (2016) signifying the preference of the ITG-B model in risk measurement (25.02 is the reported VaR estimate for the mixture of Burr models in Table 3 of Miljkovic and Grün (2016)). The second lowest relative difference is given by the ITG-IPL model while the ITG-PL model shows the largest difference. 
Similar preference is found with respect to the CTE risk measure. The seven parameter ITGB model shows excellent adherence to the empirical CTE compared to others including the Burr mixture model. The six parameter ITG-PL and ITG-IPL models do not perform well with respect to the empirical estimate. But the ITG-IPL model performs better than the Burr mixture model (82.32 is the reported CTE estimate for the Burr mixture model in Table 3 of Miljkovic and Grün (2016)).

We observe that all theoretical estimates of the proposed mixture models obtained using the trimmed data approach perform better than the estimates from the complete Danish data set. In fact, this is also the case for all two component mixture models proposed by Miljkovic and Grün (2016). For instance, our estimates of 24.92 and 54.60 for VaR and CTE compare to 25.02 and 82.32 reported in Table 3 of Miljkovic and Grün (2016) for the mixture of Burr models.

Table 5: Risk measure estimation for the three best fitted mixture models.

$\begin{array}{lcc}\text { Models } & \text { VaR } & \text { CTE } \\ \text { Empirical } & 24.61 & 54.60 \\ \text { ITG-B } & 24.92 & 54.60 \\ \text { ITG-IPL } & 27.69 & 63.58 \\ \text { ITG-PL } & 35.05 & 87.59\end{array}$

Miljkovic and Grün (2016) used three initialization methods (Euclidean distance-based initialization, $k$ means and random initialization) to estimate parameters of the mixture models with identical distribution components. However, we found that the results were similar to intensive initialization. By this we mean a number of initialization combination trials for each parameter of the models resulted in optimization reaching almost zero gradient. For the complete Danish data set, we found the following NLL values for all the two component mixture models presented in Table 1 of Miljkovic and Grün (2016) (NLL values reported by Miljkovic and Grün (2016) given in brackets): 3786.473 (3786.900) for Burr, 4162.031 (4162.040) for gamma, 3833.766 (3833.802) for inverse Burr, 3965.942 (3965.949) for inverse Gaussian, 3955.784 (3955.789) for lognormal and 4304.553 (4304.567) for Weibull models. This suggests that the optimization tools used from the $\mathrm{R}$ statistical software base package are sufficient. There is no need for additional packages.

\subsection{A simulation study}

To investigate the fit of the three models reported in Table 3, we performed a simulation study as follows: i) simulate a sample of size 2489 from the fitted model; ii) refit the model to the simulated sample; iii) repeat steps i) and ii) one hundred times. Histograms of the one hundred simulated values of the parameter estimates and their standard errors for the three models are shown in Figures 3, 4 and 5. The simulated 95 percent confidence intervals for the parameters and their true standard errors are given in Table 3. Note that some of the reported parameter estimates are outside the confidence intervals, this is because of the highly skewed nature of some of the histograms. 

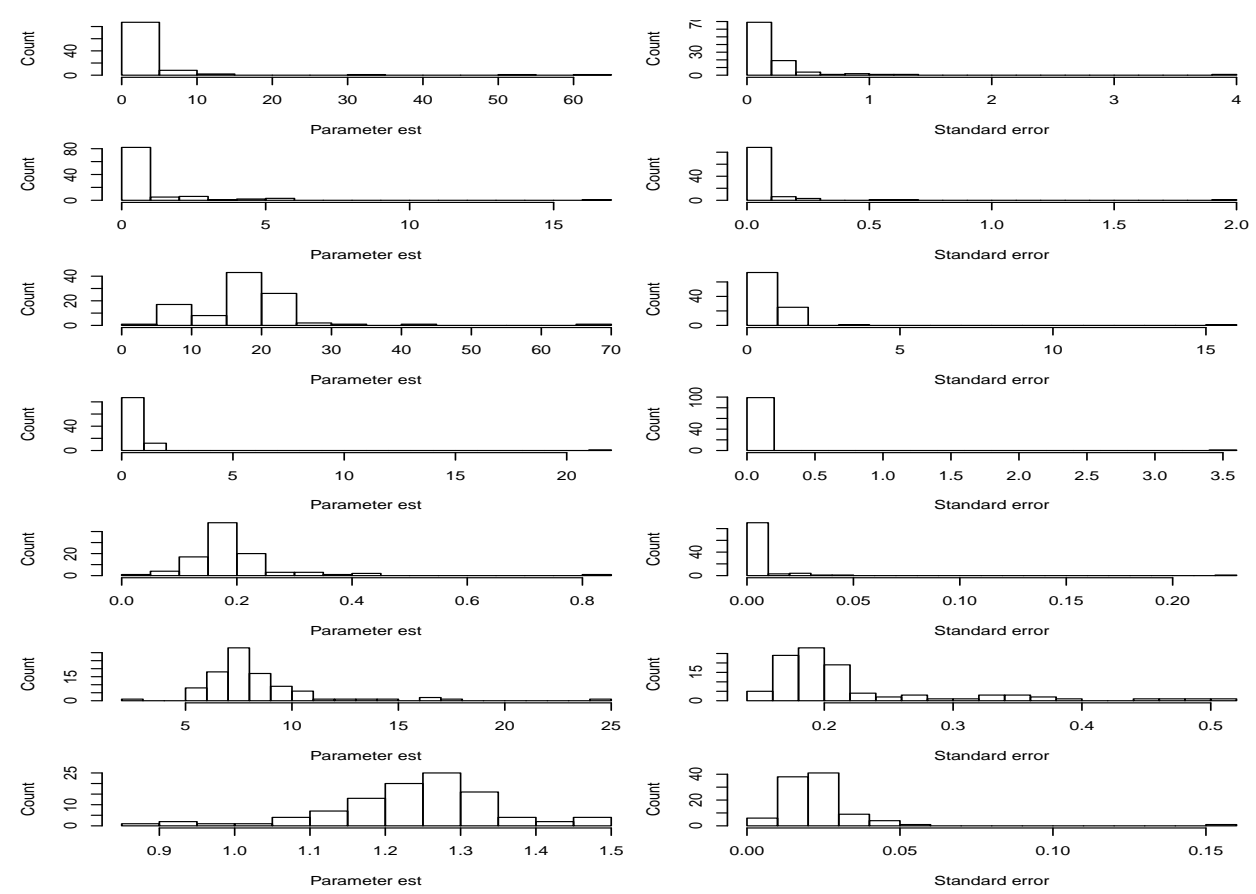

Figure 3: Histograms of the 100 simulated values of $\widehat{\phi}$, its standard error, $\widehat{\kappa}$, its standard error, $\widehat{\tau}$, its standard error, $\widehat{\theta}$, its standard error, $\widehat{\alpha}$, its standard error, $\widehat{\beta}$, its standard error, $\widehat{\lambda}$ and its standard error for the ITG-B model.
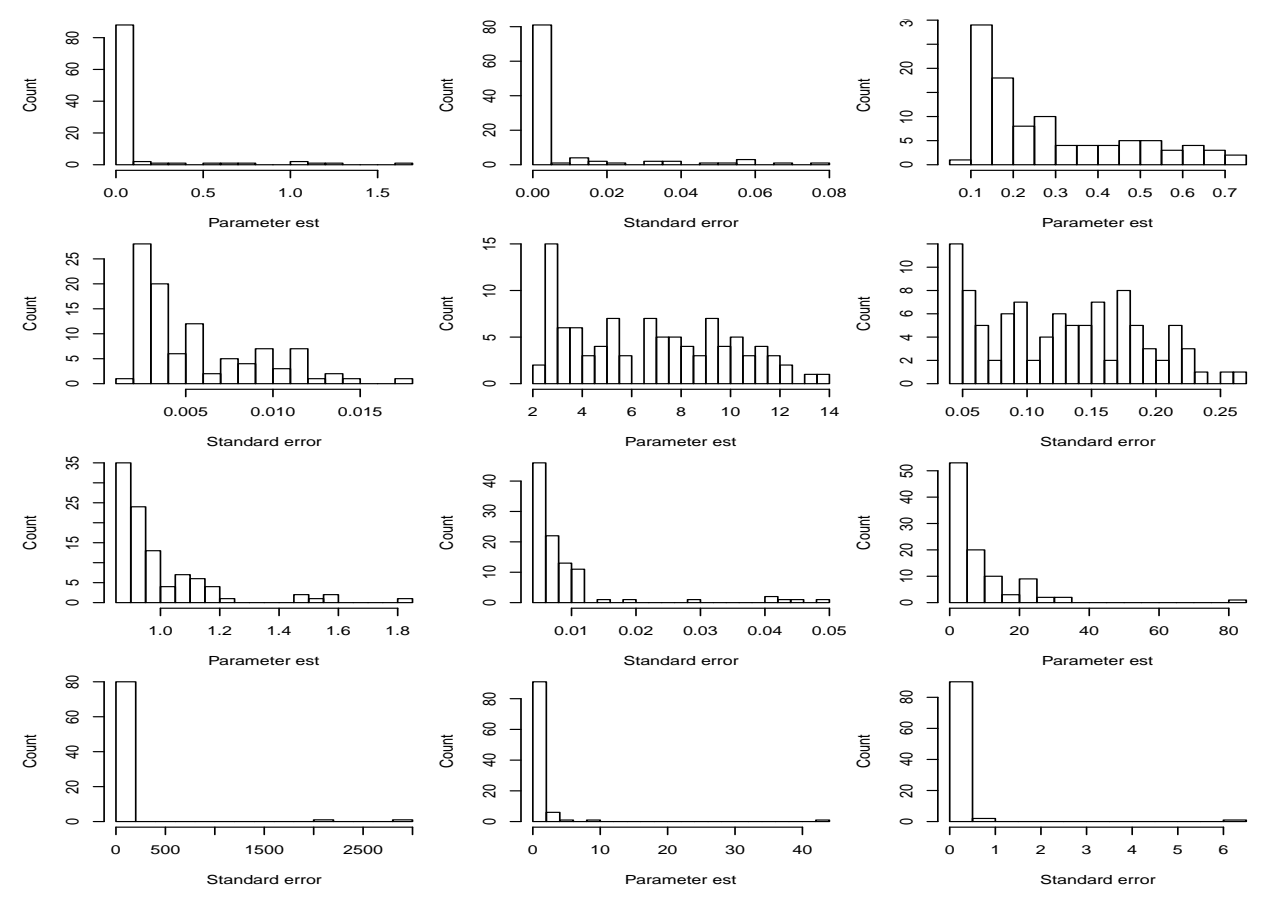

Figure 4: Histograms of the 100 simulated values of $\widehat{\phi}$, its standard error, $\widehat{\kappa}$, its standard error, $\widehat{\tau}$, its standard error, $\widehat{\theta}$, its standard error, $\widehat{\alpha}$, its standard error, $\widehat{\lambda}$ and its standard error for the ITG-IPL model. 

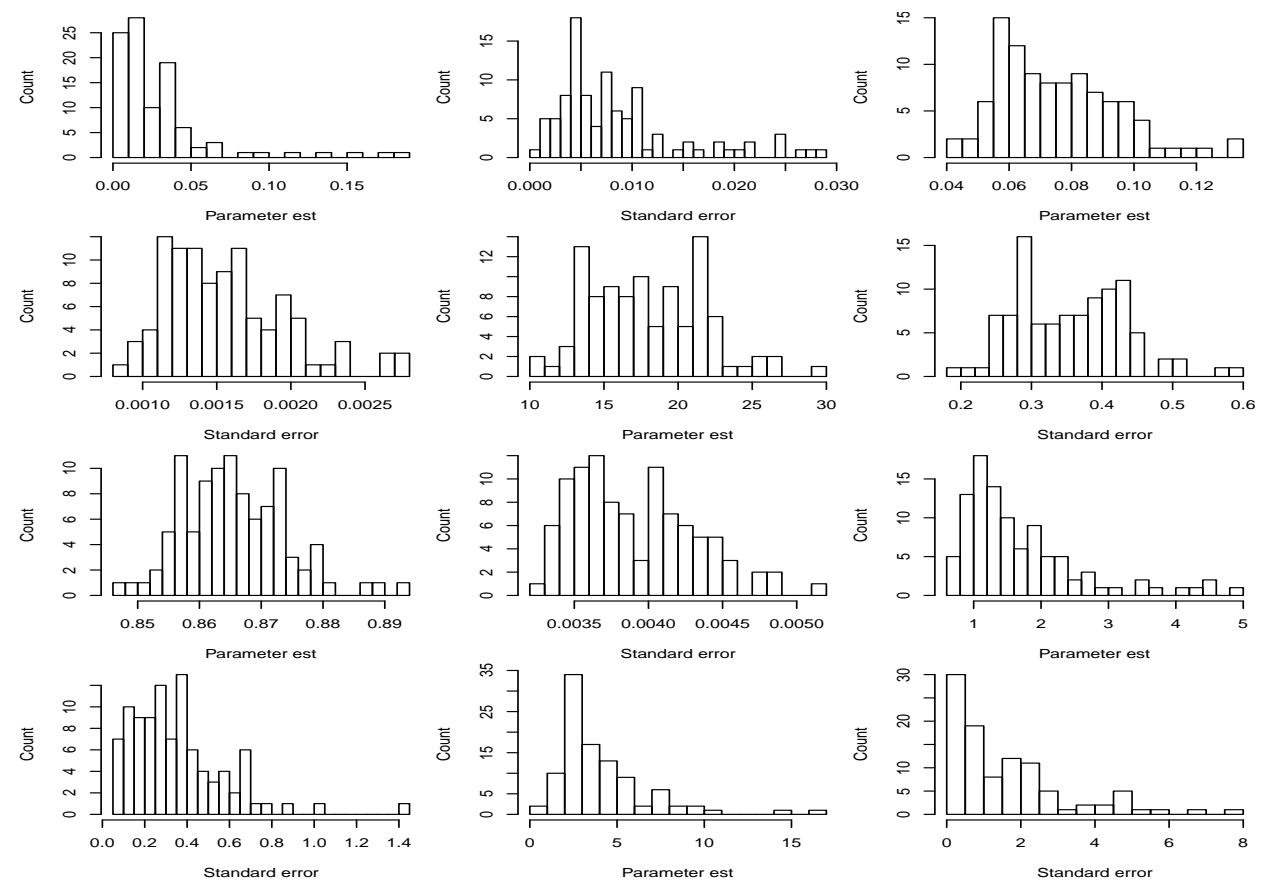

Figure 5: Histograms of the 100 simulated values of $\widehat{\phi}$, its standard error, $\widehat{\kappa}$, its standard error, $\widehat{\tau}$, its standard error, $\widehat{\theta}$, its standard error, $\widehat{\alpha}$, its standard error, $\widehat{\lambda}$ and its standard error for the ITG-PL model.

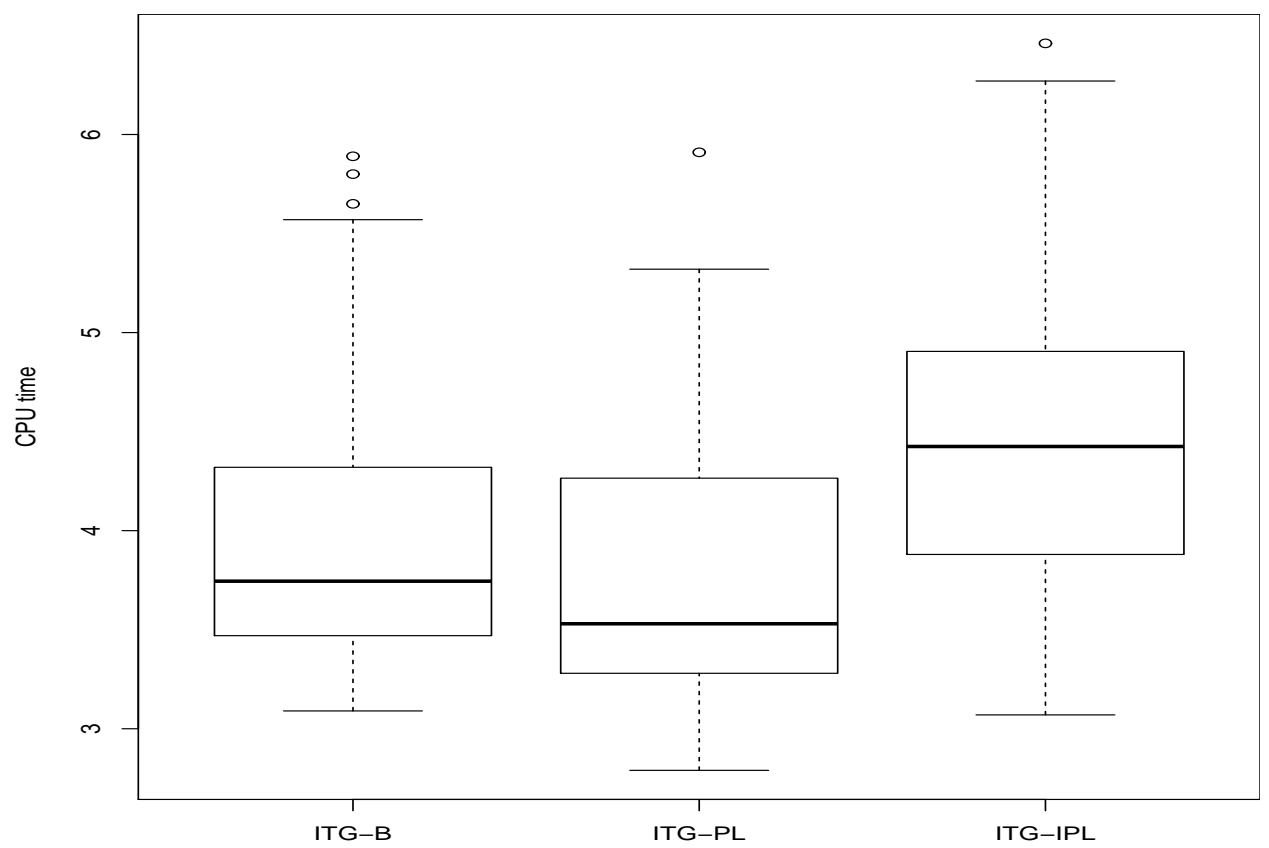

Figure 6: Boxplots of the central processing unit times taken to fit the three models 100 times.

For the ITG-B model, the distributions of most of the parameter estimates and standard errors appear skewed to the left. The distributions of $\widehat{\phi}$, its standard error, $\widehat{\kappa}$, its standard error, $\widehat{\tau}$, its 
standard error, $\widehat{\theta}$, its standard error, $\widehat{\alpha}$ and its standard error appear highly skewed to the left. The distribution of $\hat{\lambda}$ appears skewed to the right. The confidence interval for $\phi$ suggests that a 95 percent confidence interval for the weight is $(0.044,0.758)$.

For the ITG-IPL model, the distributions of all the parameter estimates and standard errors appear skewed to the left. The distributions of $\widehat{\phi}$, its standard error, standard error of $\widehat{\alpha}, \widehat{\lambda}$ and its standard error appear highly skewed to the left. The confidence interval for $\phi$ suggests that a 95 percent confidence interval for the weight is $(0.478,1)$. That is, most of the weight is given to the inverse transformed gamma distributed component.

For the ITG-PL model, the distributions of most of the parameter estimates and standard errors appear skewed to the left. The distribution of $\widehat{\tau}$ appears nearly symmetric. The distribution of its standard error appears skewed to the right. The confidence interval for $\phi$ suggests that a 95 percent confidence interval for the weight is $(0.874,0.997)$. That is, nearly all the weight is given to the inverse transformed gamma distributed component.

The central processing unit times taken to fit the three models one hundred times are shown in Figure 6. It shows that the ITG-PL model is the most time efficient, followed by the ITG-B model and then the ITG-IPL model. But the ITG-PL model did not give an adequate fit at five percent level of significance. The ITG-B model in addition to providing a better fit than the ITG-IPL model is also more time efficient.

\subsection{Sensitivity analysis}

The models fitted so far are based on the trimming condition (2). The lower limit in (2) was chosen arbitrarily as -1 . Is this an appropriate choice? We answer this question by means of a sensitivity analysis. We refitted the models in Table 3 by replacing (2) by

$$
\ln \left[\frac{x_{i}}{\max (\Psi)}\right]>d
$$

with $d=-6,-5.5, \ldots, 0$. The models in Table 3 were refitted for each value of $d$. The standard errors of the ITG-B model versus $d$ are plotted in Figure 7. The standard errors of the ITG-IPL model versus $d$ are plotted in Figure 8. The standard errors of the ITG-PL model versus $d$ are plotted in Figure 9. 

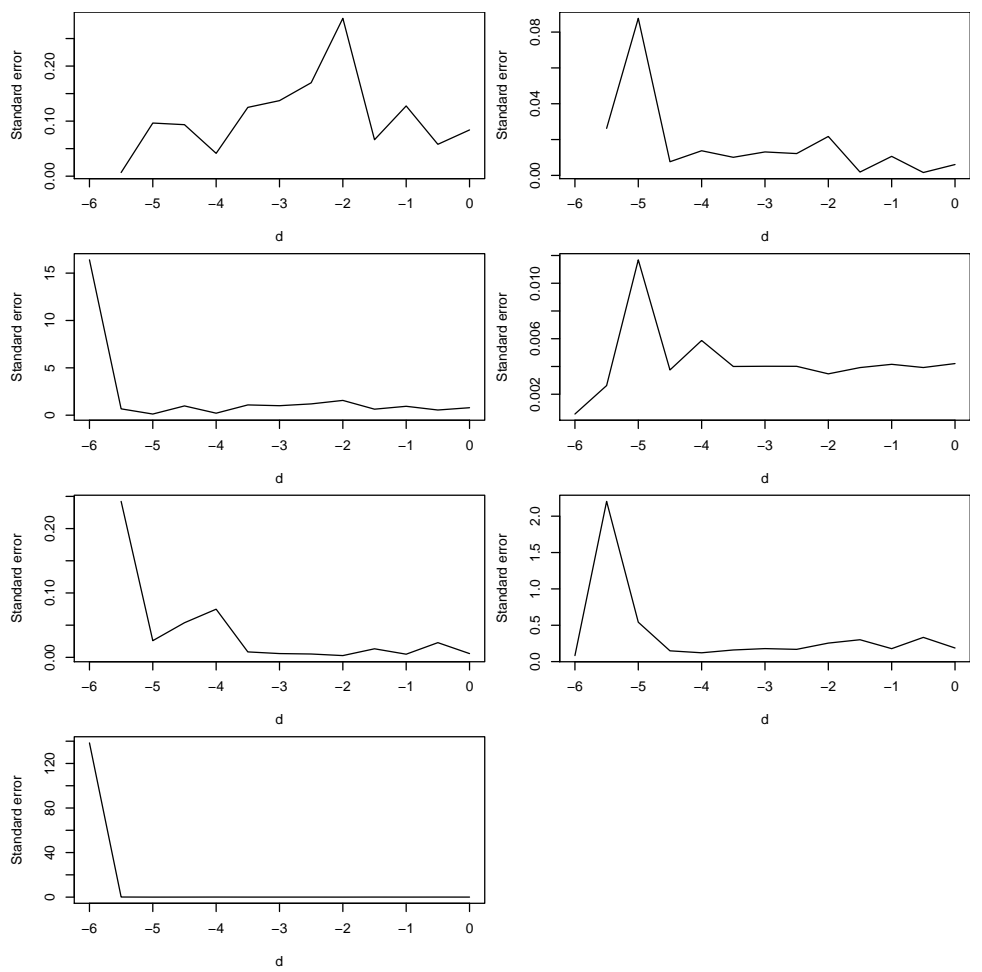

Figure 7: The standard errors of the seven parameters of the ITG-B model versus $d$.
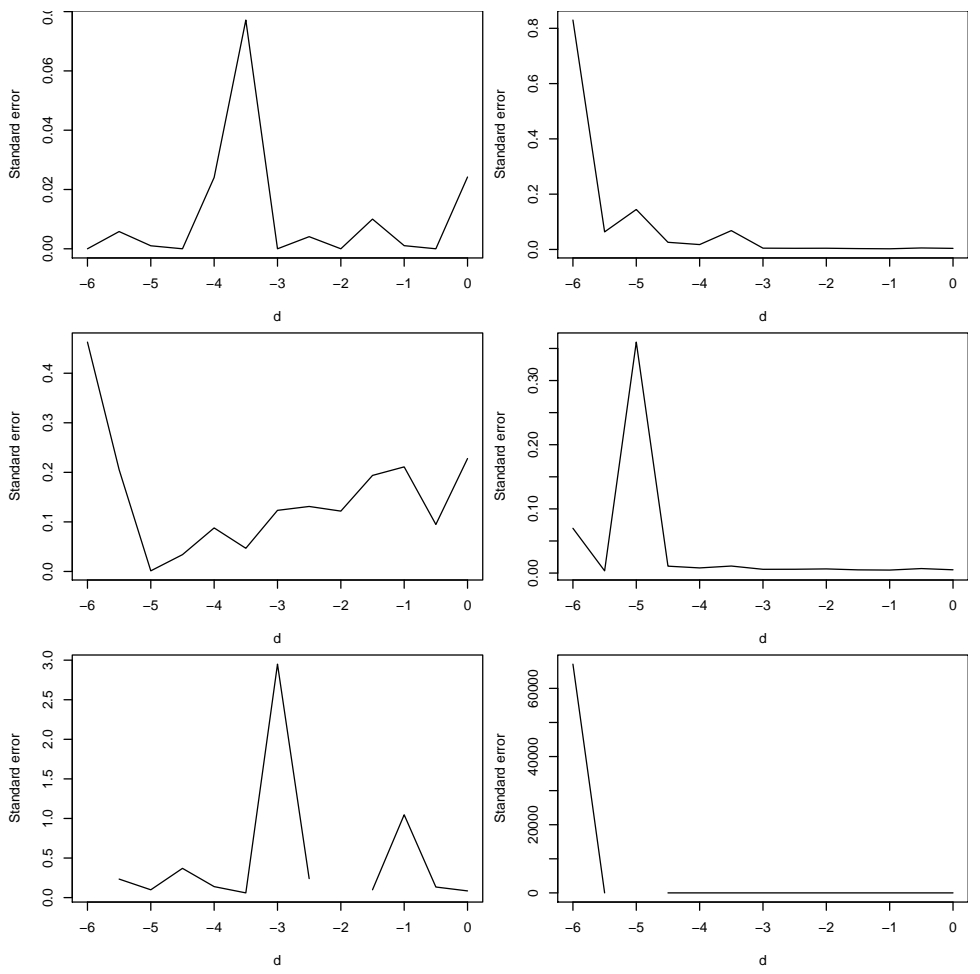

Figure 8: The standard errors of the six parameters of the ITG-IPL model versus $d$. 

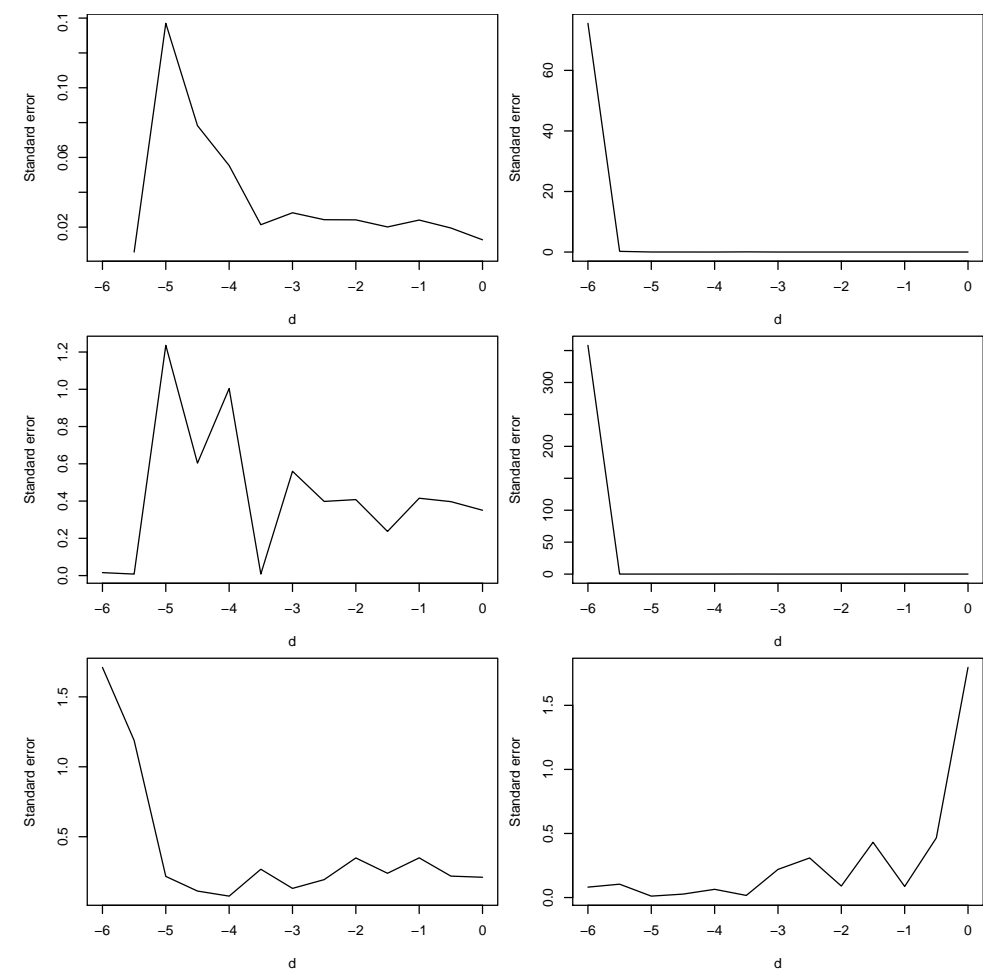

Figure 9: The standard errors of the six parameters of the ITG-PL model versus $d$.

For the ITG-B model, with the exception of two of the parameters $(\phi$ and $\theta)$, the standard errors appear small or even smallest for $d=-1$. For the ITG-IPL model, with the exception of one of the parameters $(\tau)$, the standard errors appear small or even smallest for $d=-1$. For the ITG-PL model, with the exception of one of the parameters $(\tau)$, the standard errors appear small or even smallest for $d=-1$. Hence, the choice of $d=-1$ is reasonable.

Note that $d=0$ corresponds to the untrimmed case. In many cases, $d=-1$ gives smaller standard errors than the untrimmed case.

\subsection{Software used}

Our numerical computations in this paper were assisted by the following $\mathrm{R}$ packages: the complete Danish data obtained from the SMPracticals package; tools to work with the two component mixture model found in the gendist package, see Bakar et al. (2016); distributions related to transformed beta and transformed gamma families obtained from the actuar package Dutang et al. (2008); the Anderson Darling test performed using the DescTools package; optimization for the maximum likelihood method performed using the optim function in the $\mathrm{R}$ base package. All of the $\mathrm{R}$ codes used are given in the appendix.

\section{Conclusions}

Seventeen mixture models based on the transformed beta and transformed gamma families were proposed in this paper and their performances assessed with respect to data fitting and risk esti- 
mation. Three models were found to provide better fits than the others when applied to the Danish fire loss data. These models including the mixture of the inverse transformed gamma-Burr, inverse transformed gamma-inverse paralogistic and inverse transformed gamma-paralogistic models also outperformed the best fitting models found in the recent literature in terms of goodness-of-fit.

The inverse transformed gamma-Burr model consistently showed the closest adherence to the empirical estimate of risk measures. This is shown for at least two risk measures, the Value-at-Risk and the Conditional Tail Expectation. Parameter estimation was performed by a data trimming approach and maximum likelihood estimation. An interesting finding here is the improvement in risk estimation for theoretical risk measures estimated from these parameters compared to risk estimation made from parameters estimated from the complete data (this is shown for the mixture of Burr models).

Furthermore, the two component mixture models proposed in this paper are flexible with their shapes and potentially useful in modeling many structures in practice. They were shown to represent decreasing, unimodal, bimodal and multimodal shapes with heavy tails.

The results from this paper can be extended in many ways: to provide comparisons with Bayesian estimation approaches for two component mixture models; demonstrate applications to a variety of other loss data or other areas with similar data features; allow for assessment of different combinations of mixture models; identify possible association between the goodness-of-fit and tail estimation of risk measures.

\section{Acknowledgments}

This work was supported by the Ministry of Higher Education, Malaysia under Fundamental Research Grant Scheme (FRGS) FP040-2017A. Both authors would like to thank the Editor and the two referees for careful reading and comments which greatly improved the paper.

\section{References}

Ahn, S., Kim, J. H., Ramaswami, V., 2012. A new class of models for heavy tailed distributions in finance and insurance risk. Insurance: Mathematics and Economics 51 (1), 43-52.

Asgharzadeh, A., Nadarajah, S., Sharafi, F., 2016. Generalized inverse Lindley distribution with application to Danish fire insurance data. Communications in Statistics-Theory and Methods (justaccepted).

Bakar, S. A., Hamzah, N., Maghsoudi, M., Nadarajah, S., 2015. Modeling loss data using composite models. Insurance: Mathematics and Economics 61, 146-154.

Bakar, S. A. A., Nadarajah, S., Adzhar, Z. A. A. K., Mohamed, I., 2016. Gendist: An R package for generated probability distribution models. PLoS One 11 (6), e0156537.

Bernardi, M., Maruotti, A., Petrella, L., 2012. Skew mixture models for loss distributions: A Bayesian approach. Insurance: Mathematics and Economics 51 (3), 617-623.

Brazauskas, V., Kleefeld, A., 2009. Robust and efficient fitting of the generalized pareto distribution with actuarial applications in view. Insurance: Mathematics and Economics 45 (3), 424-435. 
Calderín-Ojeda, E., 2015. On the composite weibull-burr model to describe claim data. Communication in Statistics: Case Studies, Data Analysis and Applications 1, 59-69.

Cooray, K., Ananda, M. M., 2005. Modeling actuarial data with a composite lognormal-Pareto model. Scandinavian Actuarial Journal 2005 (5), 321-334.

Dutang, C., Goulet, V., Pigeon, M., 2008. actuar: An R package for actuarial science. Journal of Statistical Software $25(7), 38$.

URL http://www.jstatsoft.org/v25/i07

Eling, M., 2012. Fitting insurance claims to skewed distributions: Are the skew-normal and skewstudent good models? Insurance: Mathematics and Economics 51 (2), 239-248.

Klugman, S. A., Panjer, H. H., Willmot, G. E., 2012. Loss Models: From Data to Decisions. Vol. 715. John Wiley and Sons.

Klugman, S. A., Parsa, R., 1999. Fitting bivariate loss distributions with copulas. Insurance: Mathematics and Economics 24 (1), 139-148.

Lee, S. C., Lin, X. S., 2010. Modeling and evaluating insurance losses via mixtures of Erlang distributions. North American Actuarial Journal 14 (1), 107-130.

McNeil, A. J., 1997. Estimating the tails of loss severity distributions using extreme value theory. ASTIN Bulletin 27 (01), 117-137.

Miljkovic, T., Grün, B., 2016. Modeling loss data using mixtures of distributions. Insurance: Mathematics and Economics 70, 387-396.

Nadarajah, S., Bakar, S., 2014. New composite models for the Danish fire insurance data. Scandinavian Actuarial Journal 2014 (2), 180-187.

Pigeon, M., Denuit, M., 2011. Composite lognormal-Pareto model with random threshold. Scandinavian Actuarial Journal 2011 (3), 177-192.

Resnick, S. I., 1997. Discussion of the Danish data on large fire insurance losses. Astin Bulletin 27 (01), 139-151.

Ruckdeschel, P., Horbenko, N., 2013. Optimally robust estimators in generalized Pareto models. Statistics 47 (4), 762-791.

Scollnik, D. P., 2007. On composite lognormal-Pareto models. Scandinavian Actuarial Journal 2007 (1), 20-33.

Scollnik, D. P., Sun, C., 2012. Modeling with Weibull-Pareto models. North American Actuarial Journal 16 (2), 260-272.

Venter, G., 1983. Transformed beta and gamma distributions and aggregate losses. In: Proceedings of the Casualty Actuarial Society. Vol. 70. pp. 289-308. 


\section{Appendix: $\mathrm{R}$ codes}

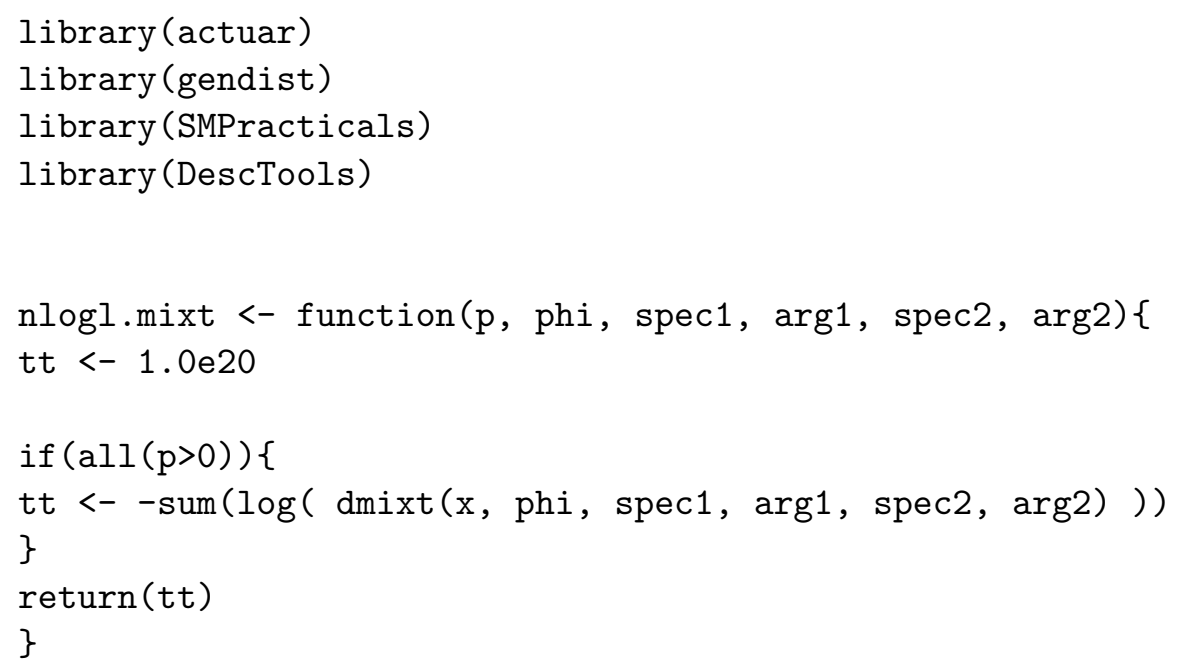




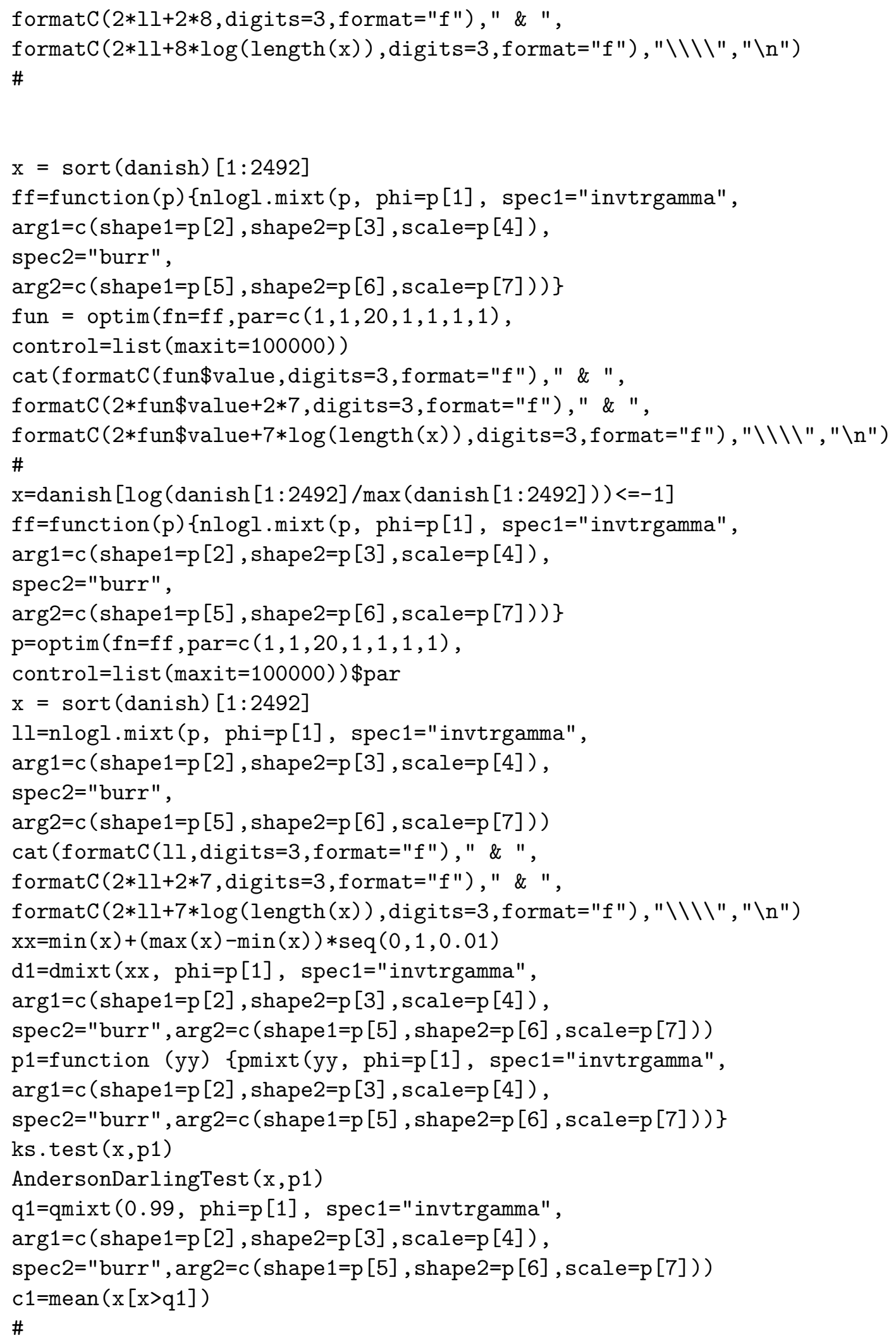




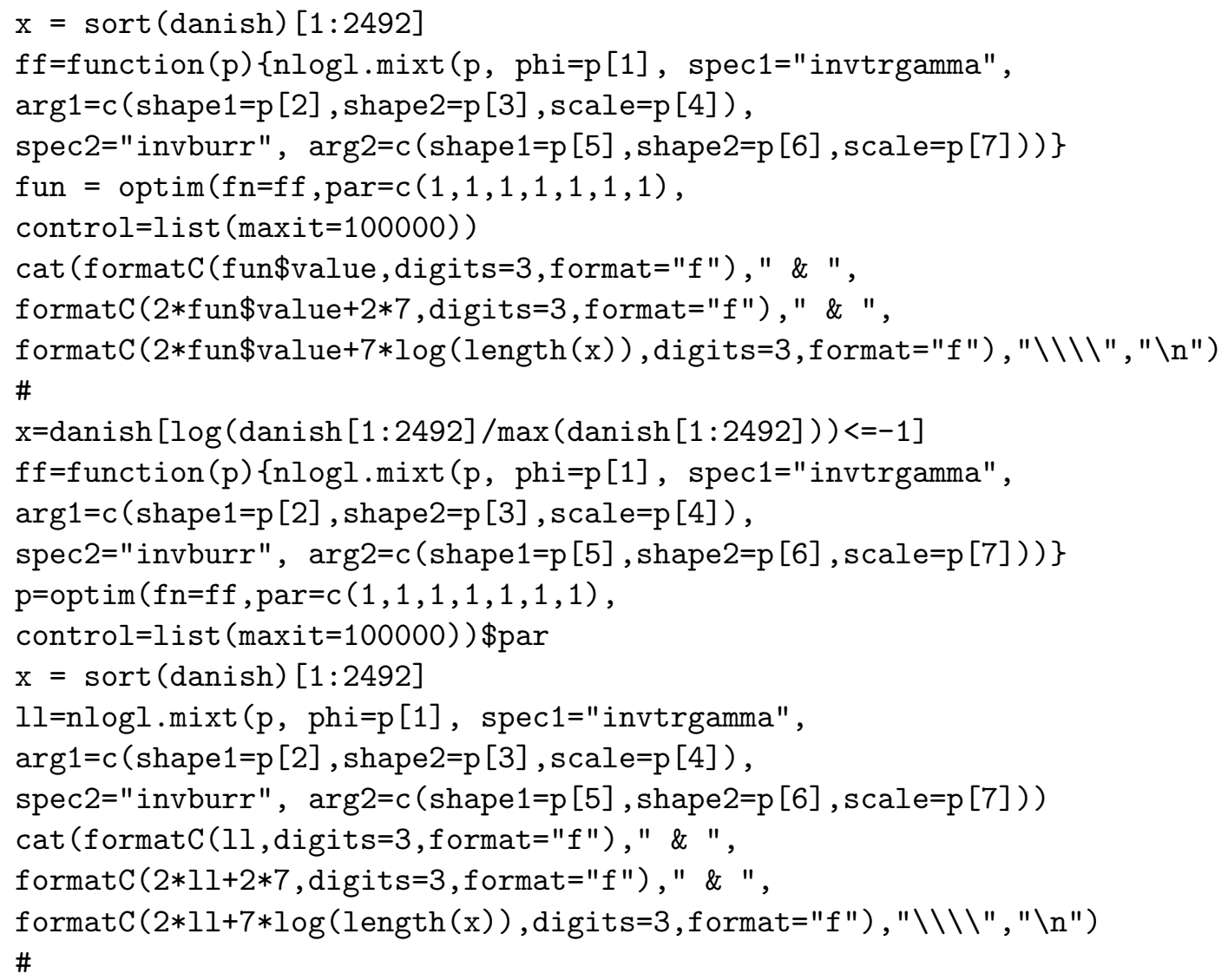




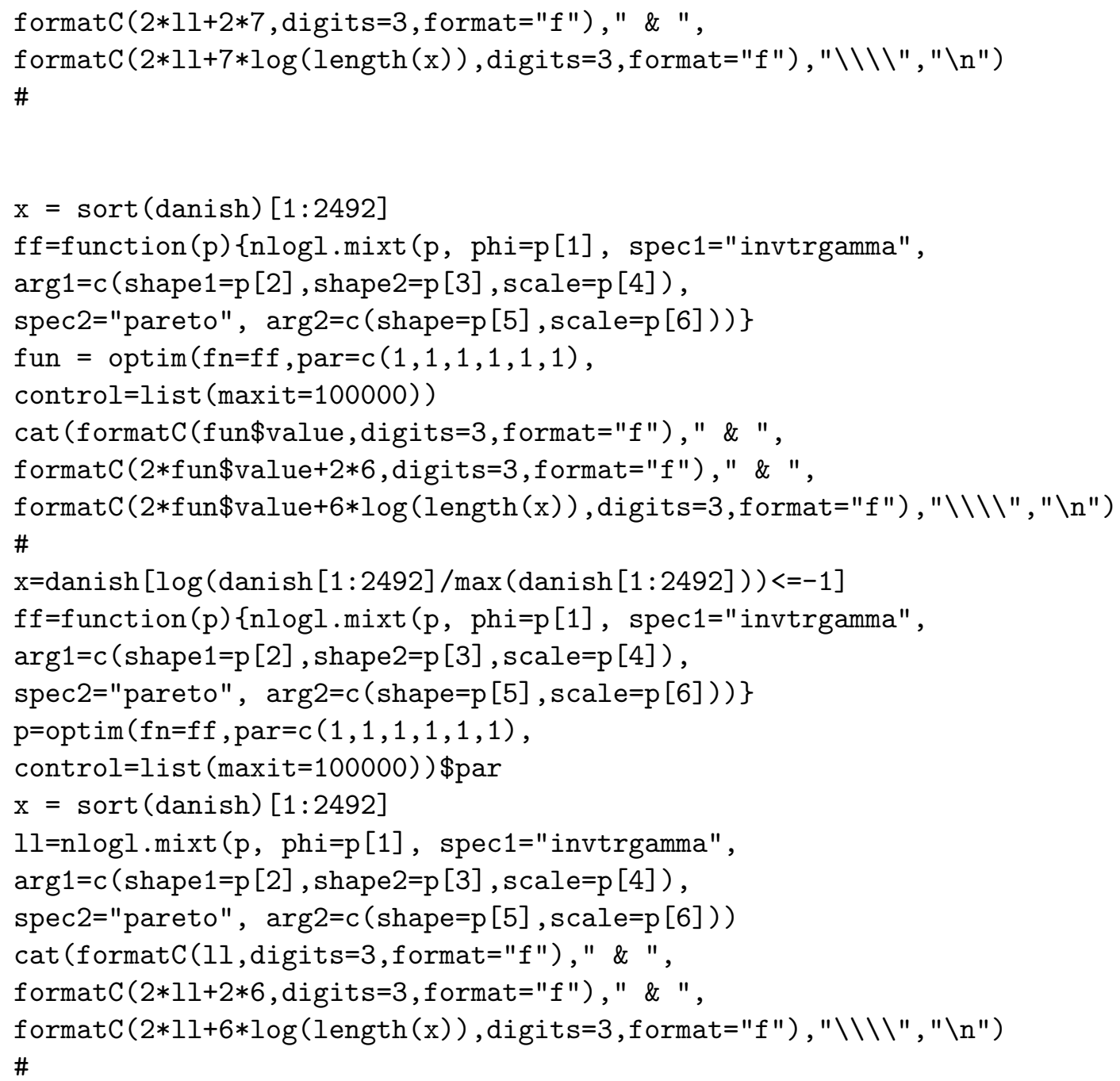




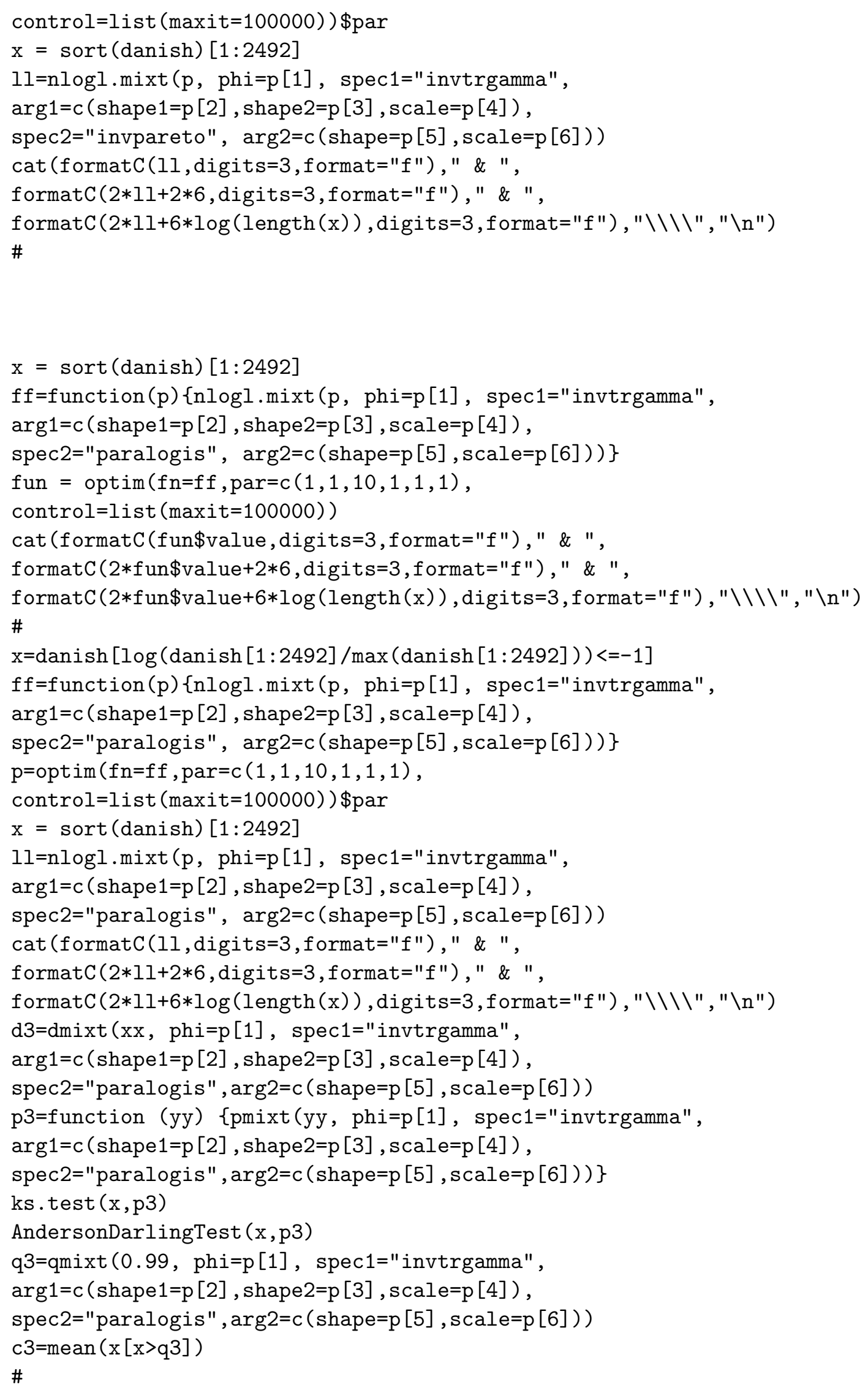




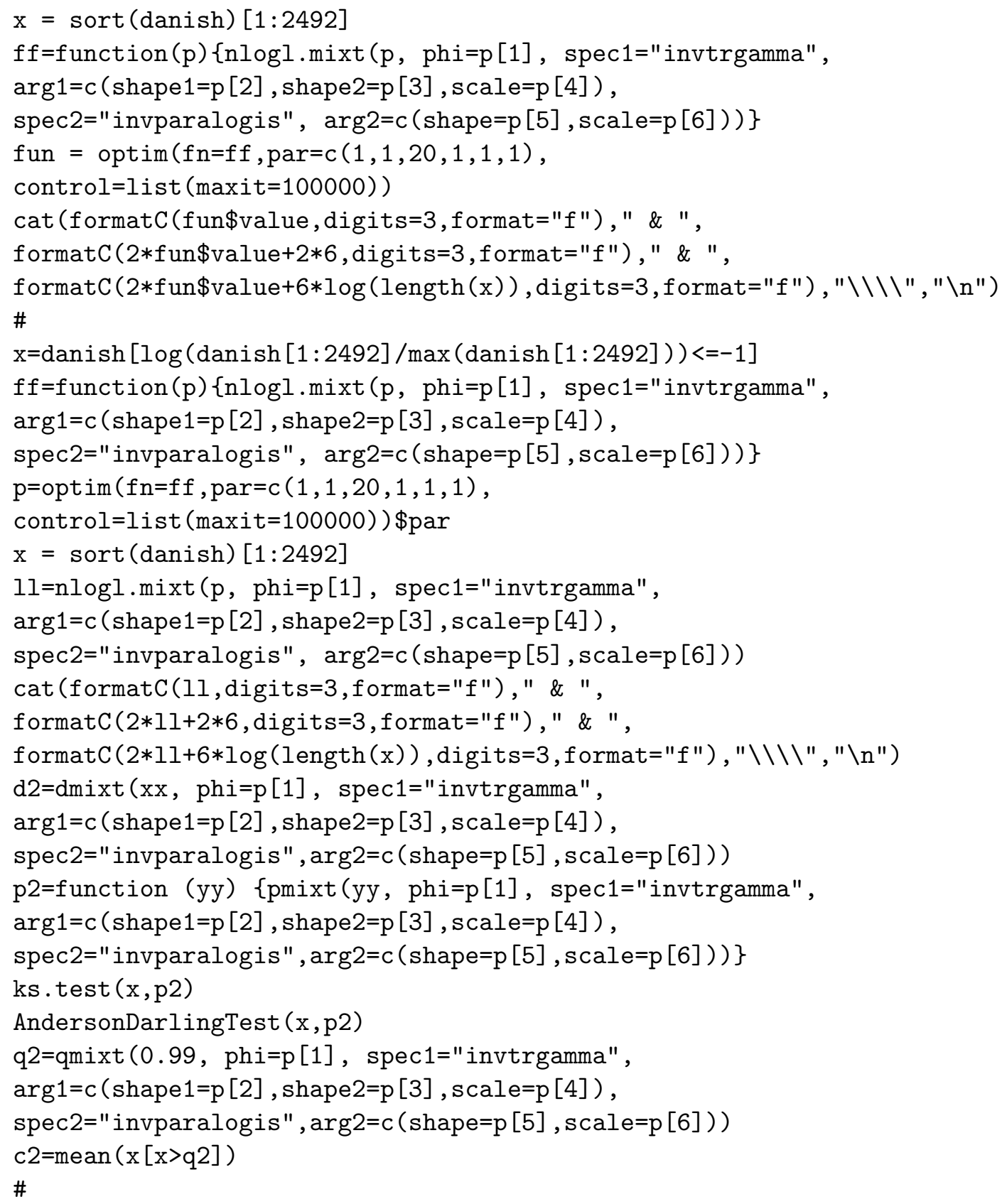




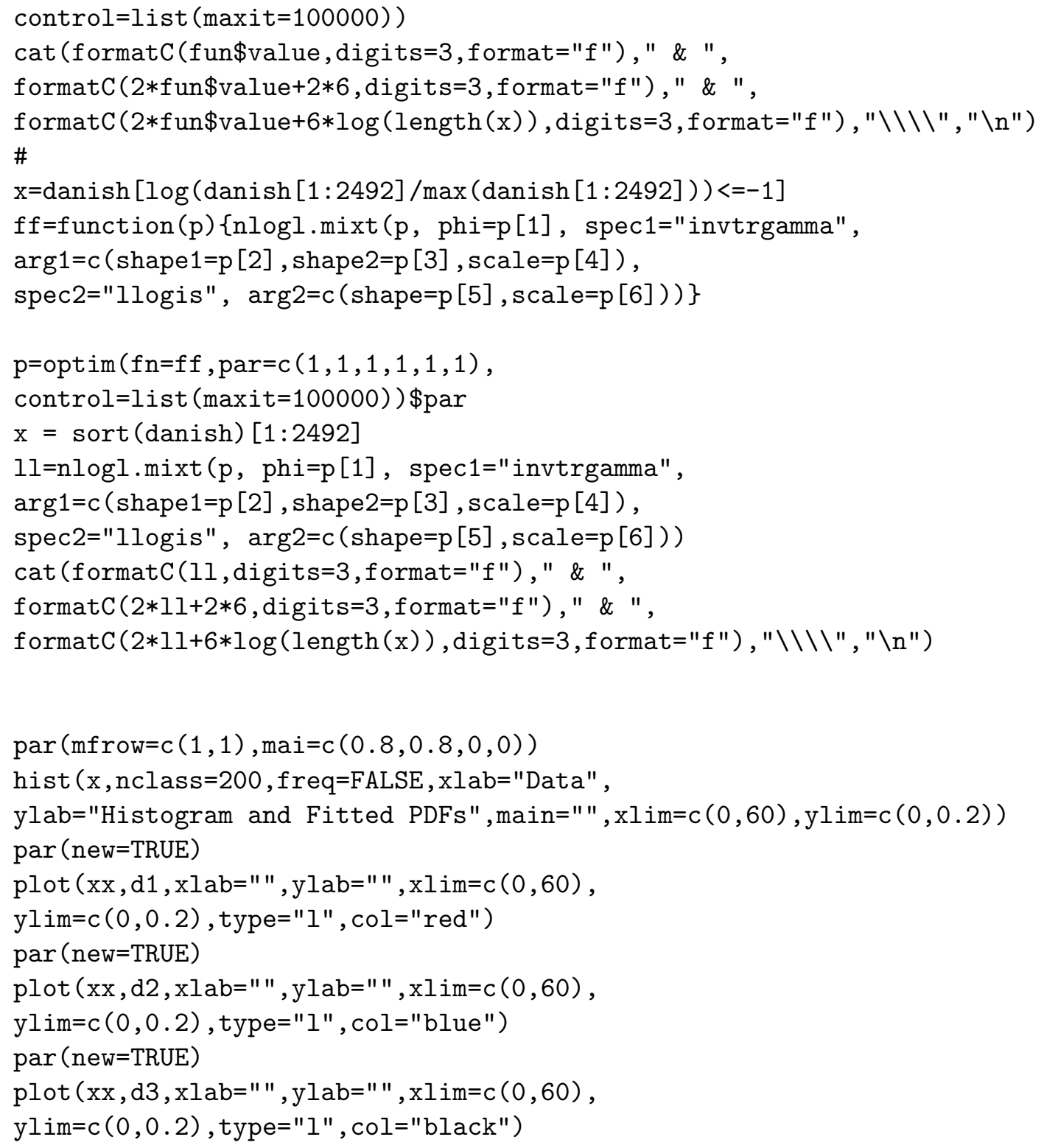




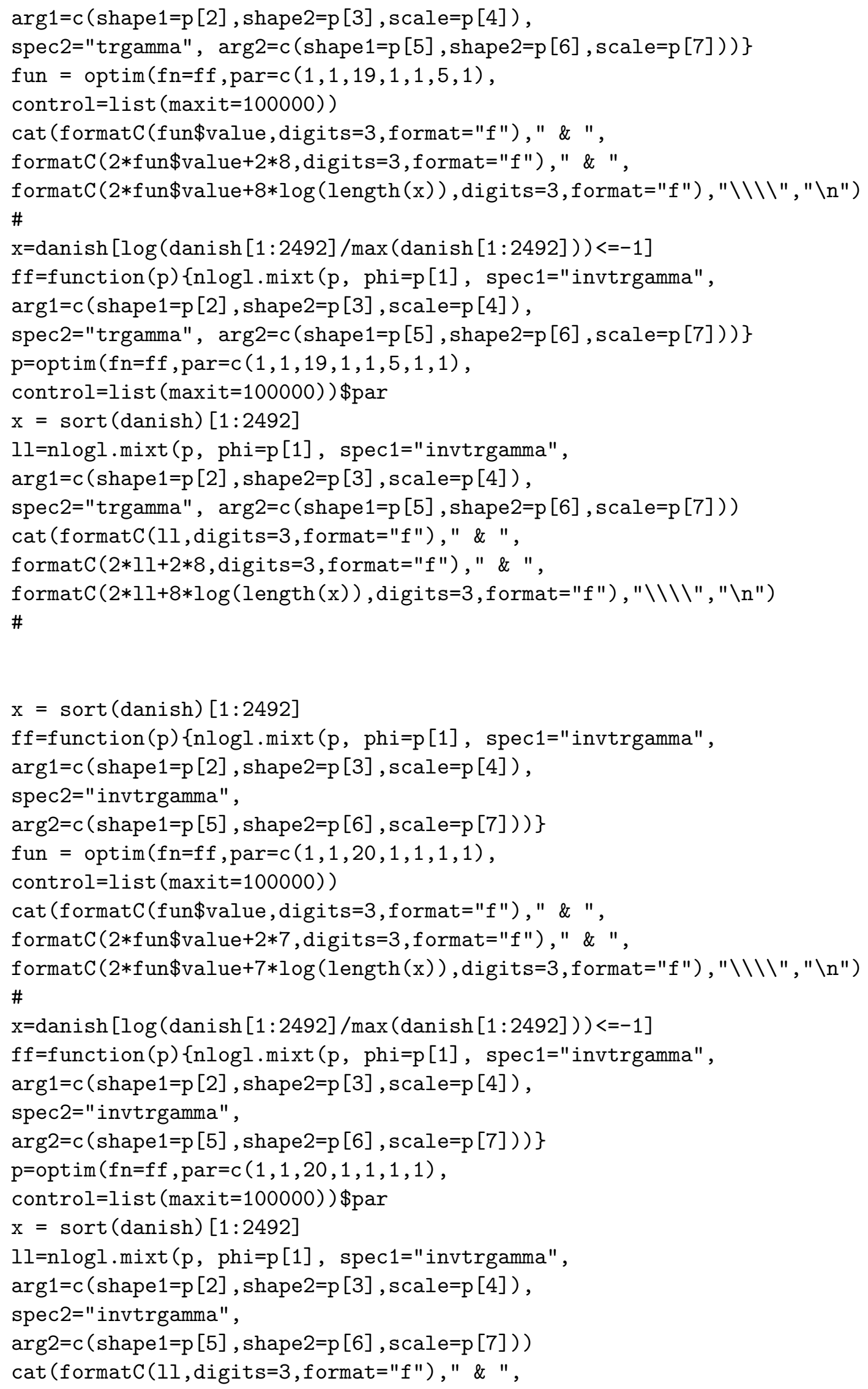




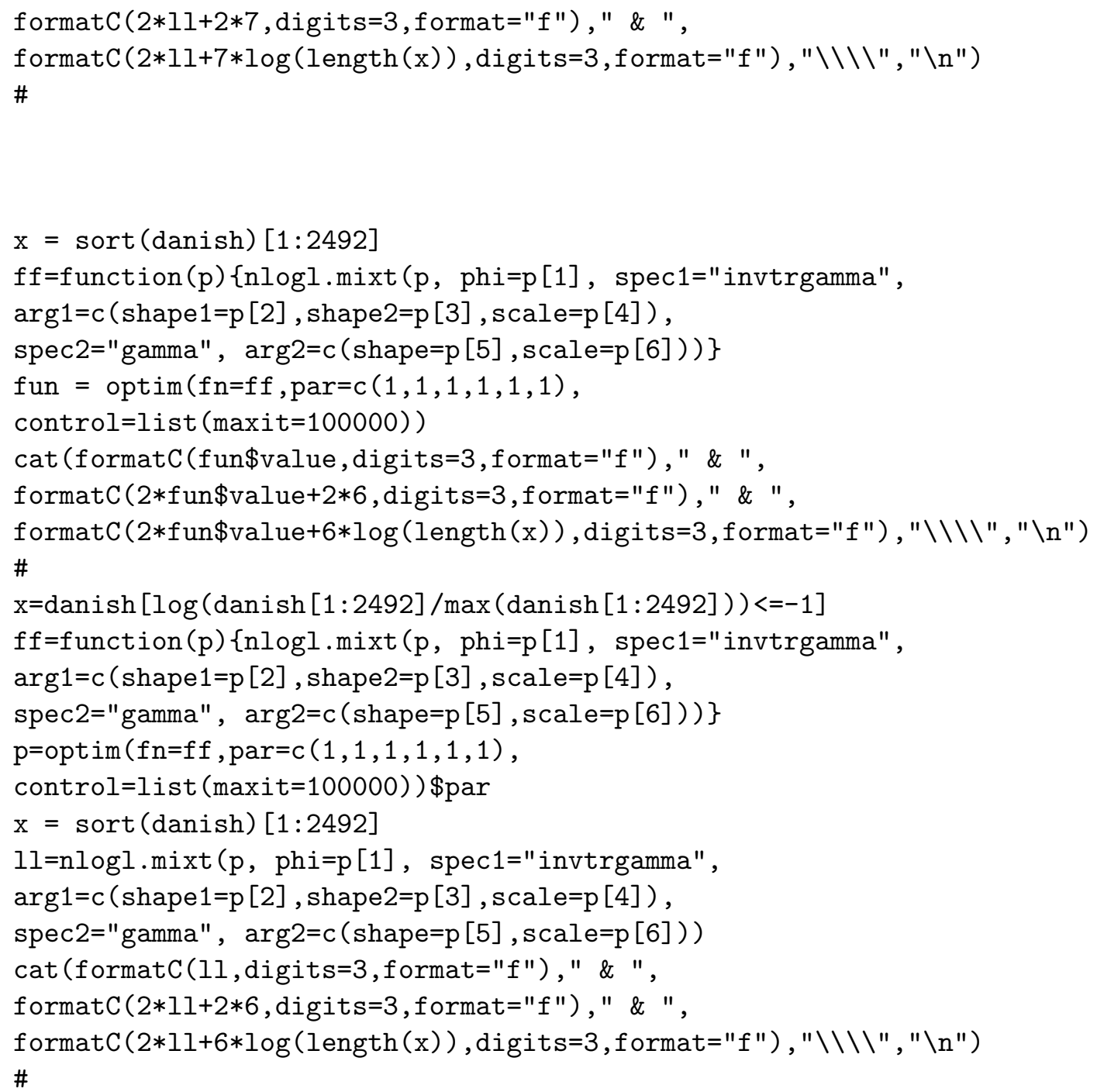




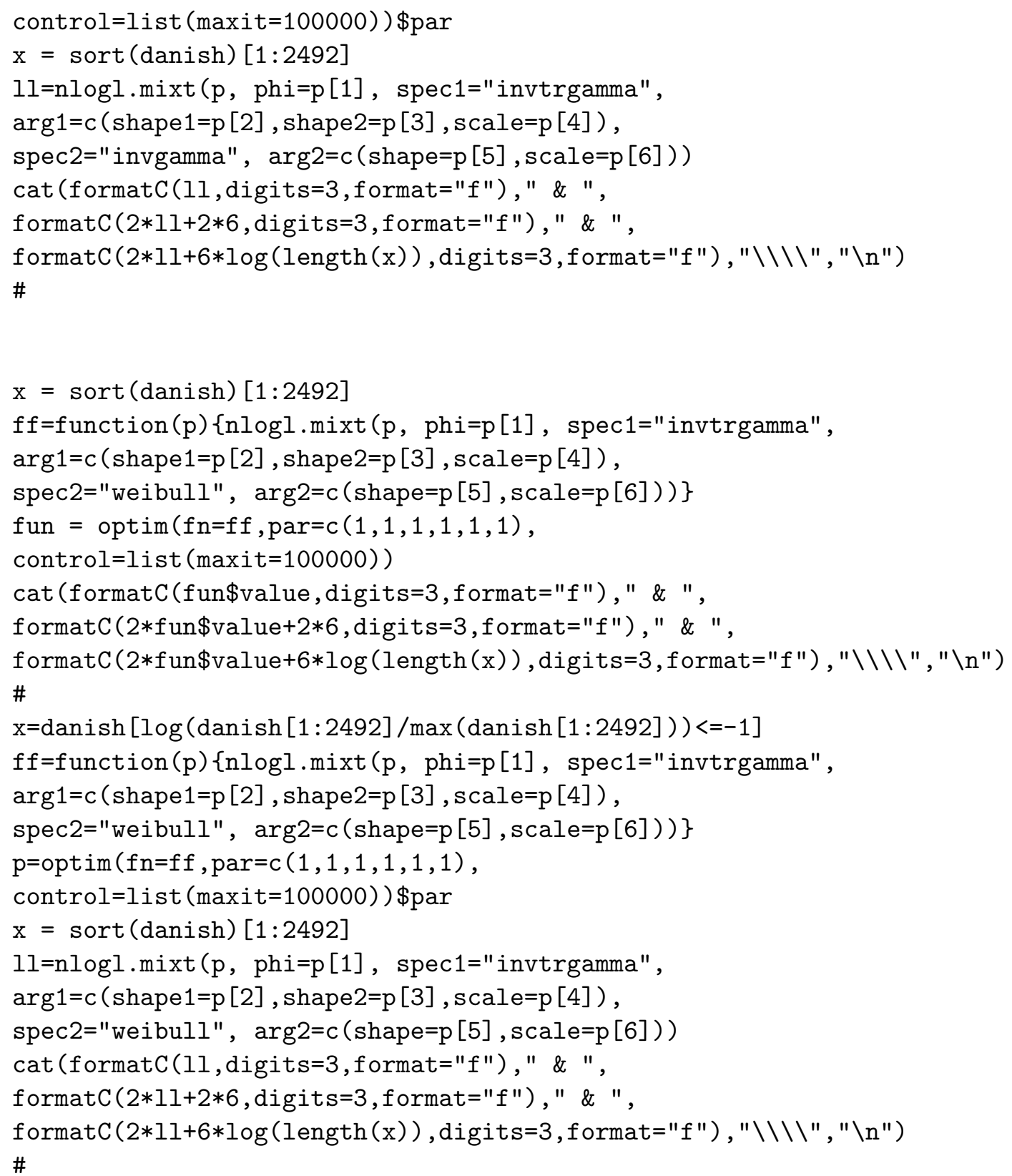




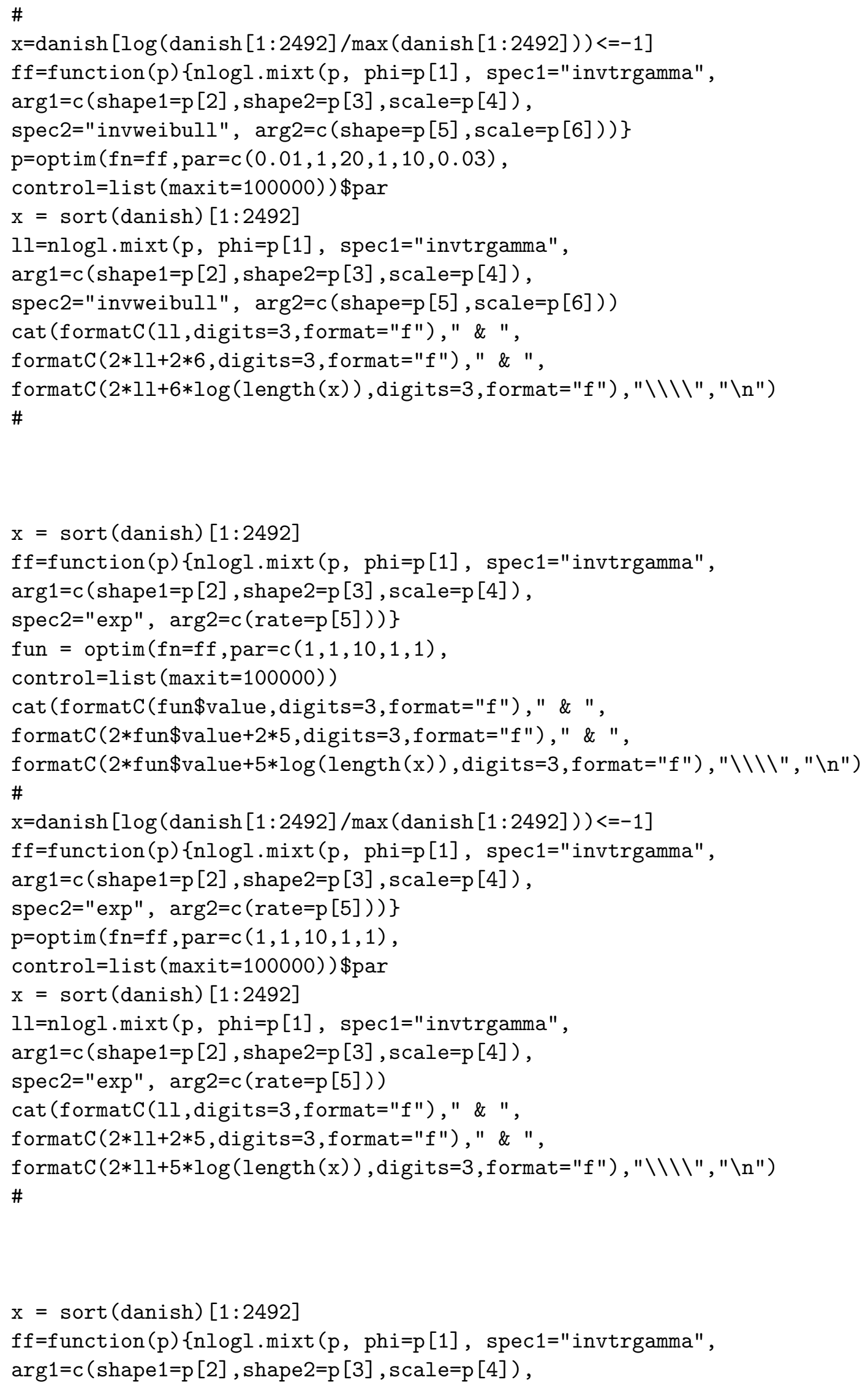




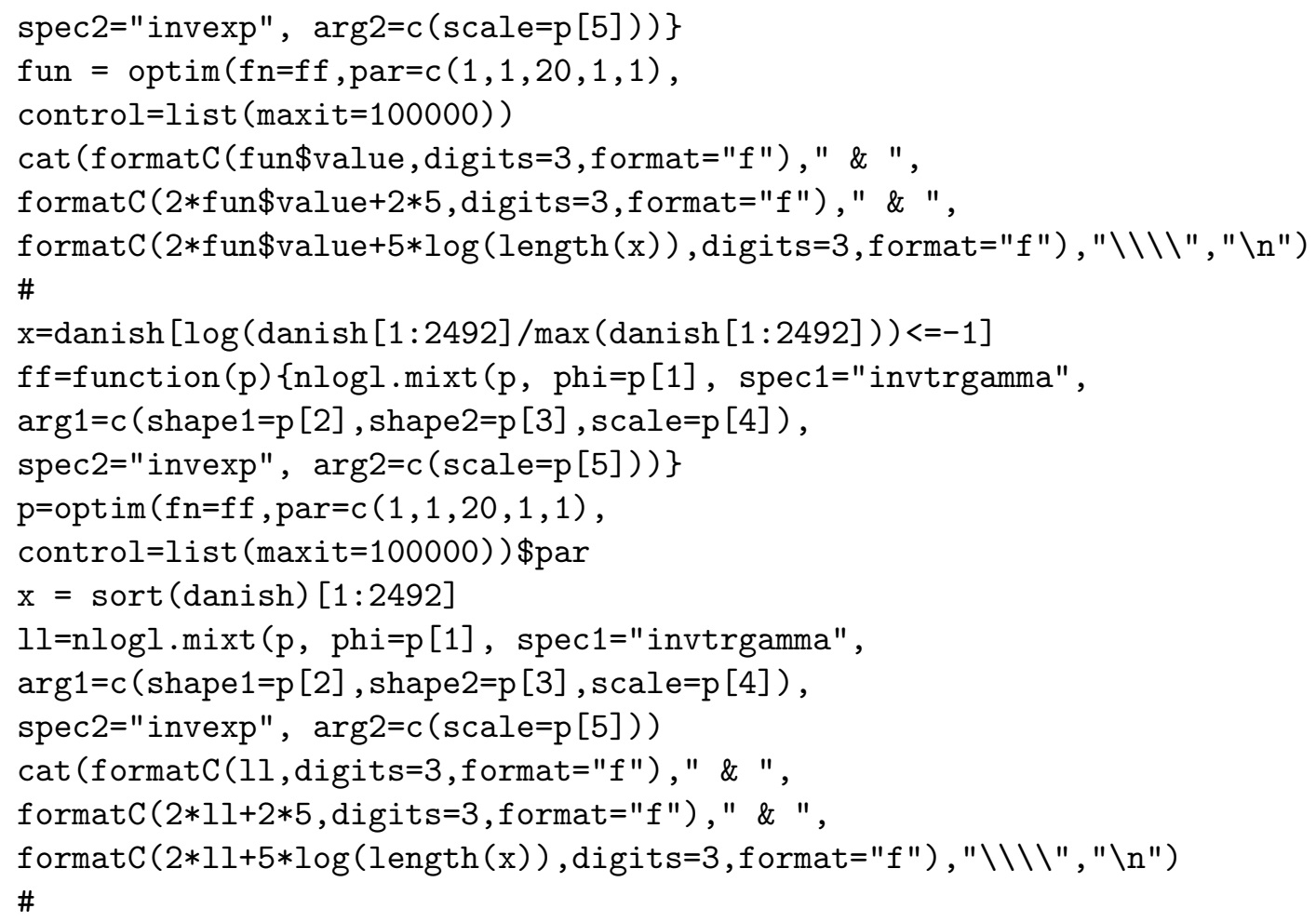


plot (seq $(-6,0$, by $=0.5)$, as numeric $(f$ se $[2]),, x l a b=" d "$, ylab="Standard error", type="1")

plot (seq $(-6,0, b y=0.5)$, as.numeric $(f s e[3]),, x l a b=" d "$, ylab="Standard error", type="1")

$\operatorname{plot}(\operatorname{seq}(-6,0, \mathrm{by}=0.5)$, as.numeric $(f \mathrm{se}[4]),, \mathrm{xlab}=" d "$, ylab="Standard error", type="l")

plot (seq $(-6,0, b y=0.5)$, as.numeric $(f$ se $[5]),, x l a b=" d "$, ylab="Standard error", type="1")

plot (seq $(-6,0$, by $=0.5)$, as numeric $(f s e[6]),, x l a b=" d "$, ylab="Standard error", type="1")

plot (seq $(-6,0, b y=0.5)$, as.numeric $(f \operatorname{se}[7]),, x l a b=" d "$, ylab="Standard error", type="l")

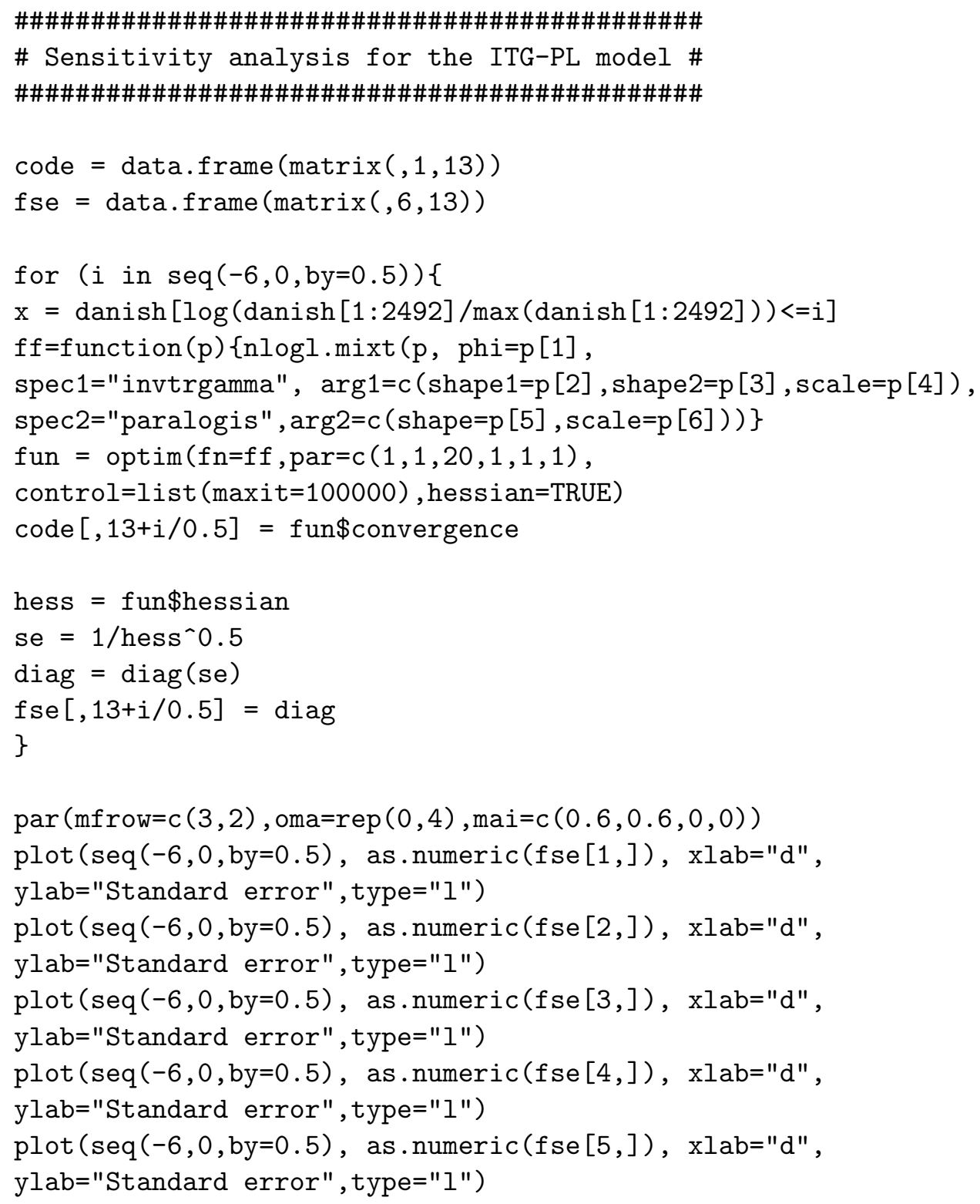


plot (seq $(-6,0, b y=0.5)$, as.numeric $(f$ se $[6]),, x l a b=" d "$, ylab="Standard error", type="1")

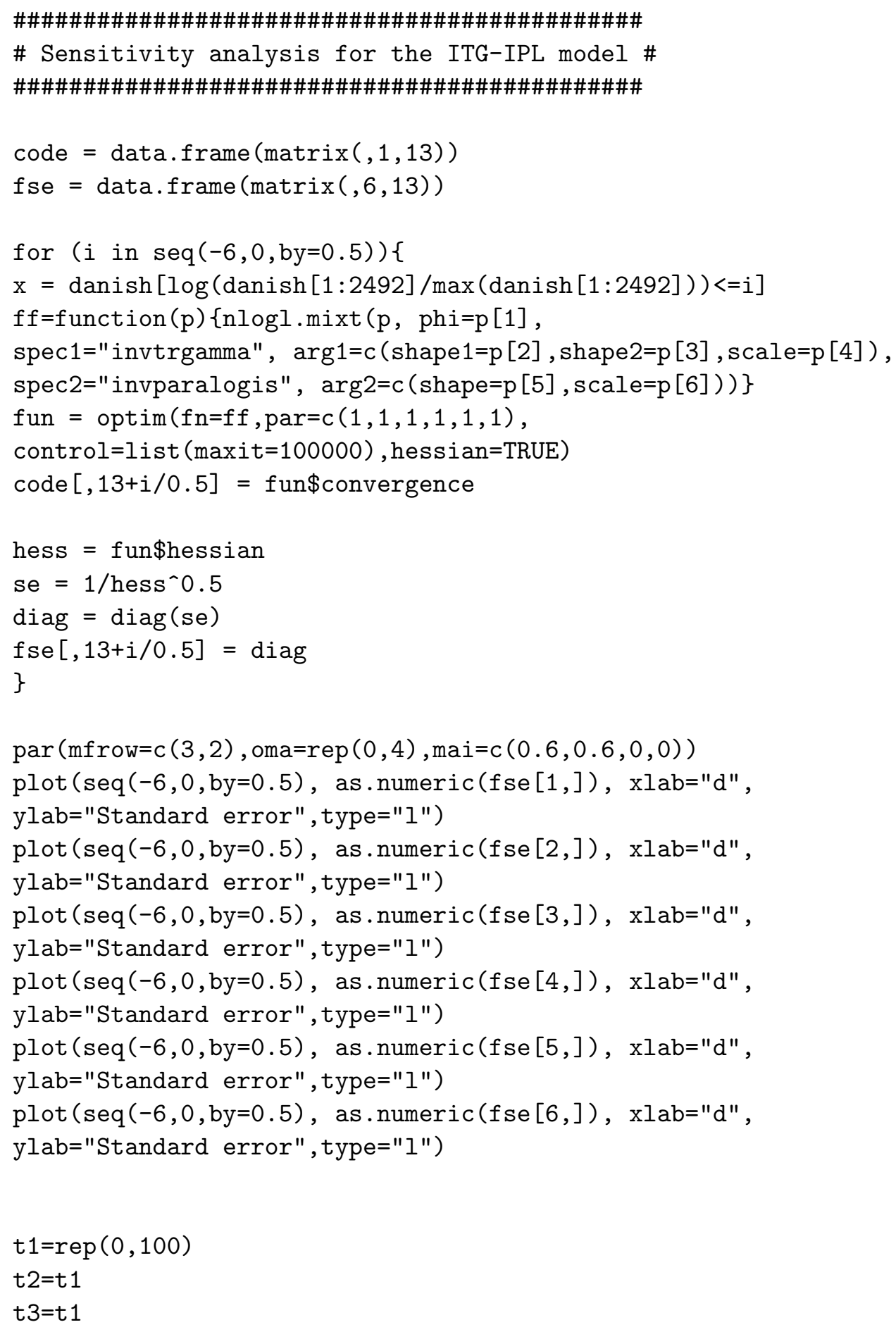


\# Simulation for the ITG-B model \#

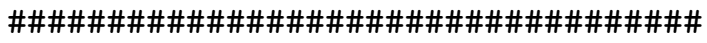

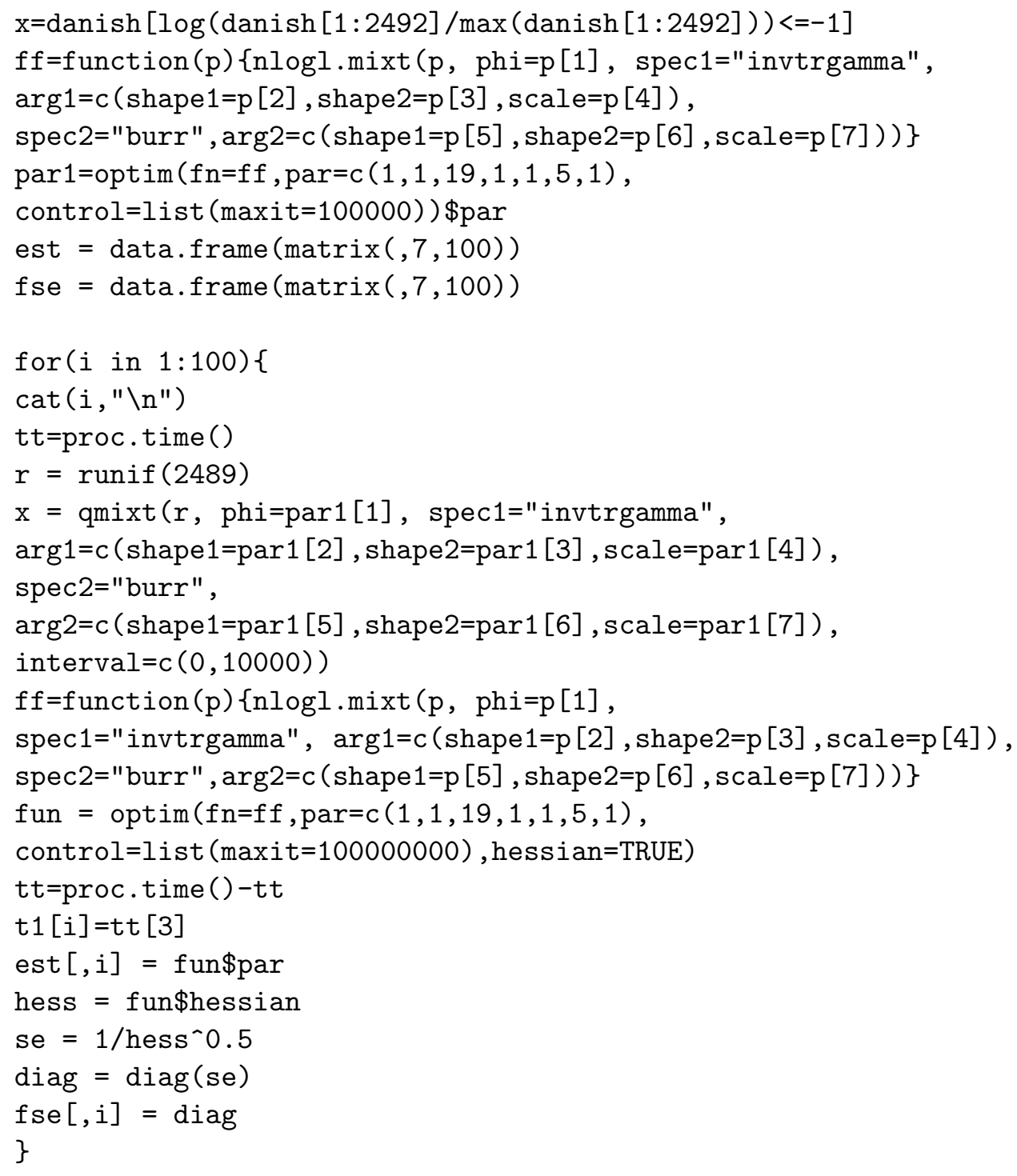




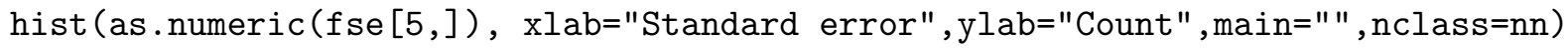
hist (as.numeric(est $[6]$,$) , xlab="Parameter est",ylab="Count", main=" ", nclass=nn)$ hist (as.numeric (fse $[6$,$] ), xlab="Standard error", ylab="Count", main=" ", nclass=nn)$ hist (as.numeric (est $[7$,$] ), \mathrm{xlab}=$ "Parameter est", ylab="Count", main=" ",nclass=nn) hist (as.numeric(fse $[7$,$] ), xlab="Standard error",ylab="Count", main=" ",nclass=nn)$

quantile(as.numeric (est $[1],), \operatorname{prob}=c(0.025,0.975))$

$\mathrm{xx}=\mathrm{as}$. numeric $(\mathrm{fse}[1]$,

quantile (xx $[$ ! is.na(xx)], prob $=c(0.025,0.975))$

quantile (as.numeric(est $[2]$,$) , prob =c(0.025,0.975)$ )

quantile (as.numeric (fse $[2]$,$) , prob=c (0.025,0.975))$

quantile(as.numeric (est $[3]$,$) , prob =c(0.025,0.975))$

quantile(as.numeric (fse $[3]$,$) , prob =c(0.025,0.975))$

quantile (as.numeric (est $[4],), \operatorname{prob}=c(0.025,0.975))$

quantile (as.numeric (fse $[4],), \operatorname{prob}=c(0.025,0.975))$

quantile (as.numeric (est $[5]$,$) , prob =c(0.025,0.975))$

quantile (as.numeric (fse $[5],), \operatorname{prob}=c(0.025,0.975))$

quantile (as.numeric (est $[6],), \operatorname{prob}=c(0.025,0.975))$

quantile(as.numeric (fse $[6],), \operatorname{prob}=c(0.025,0.975))$

quantile (as.numeric (est $[7],), \operatorname{prob}=c(0.025,0.975))$

quantile (as.numeric (fse $[7],), \operatorname{prob}=c(0.025,0.975))$

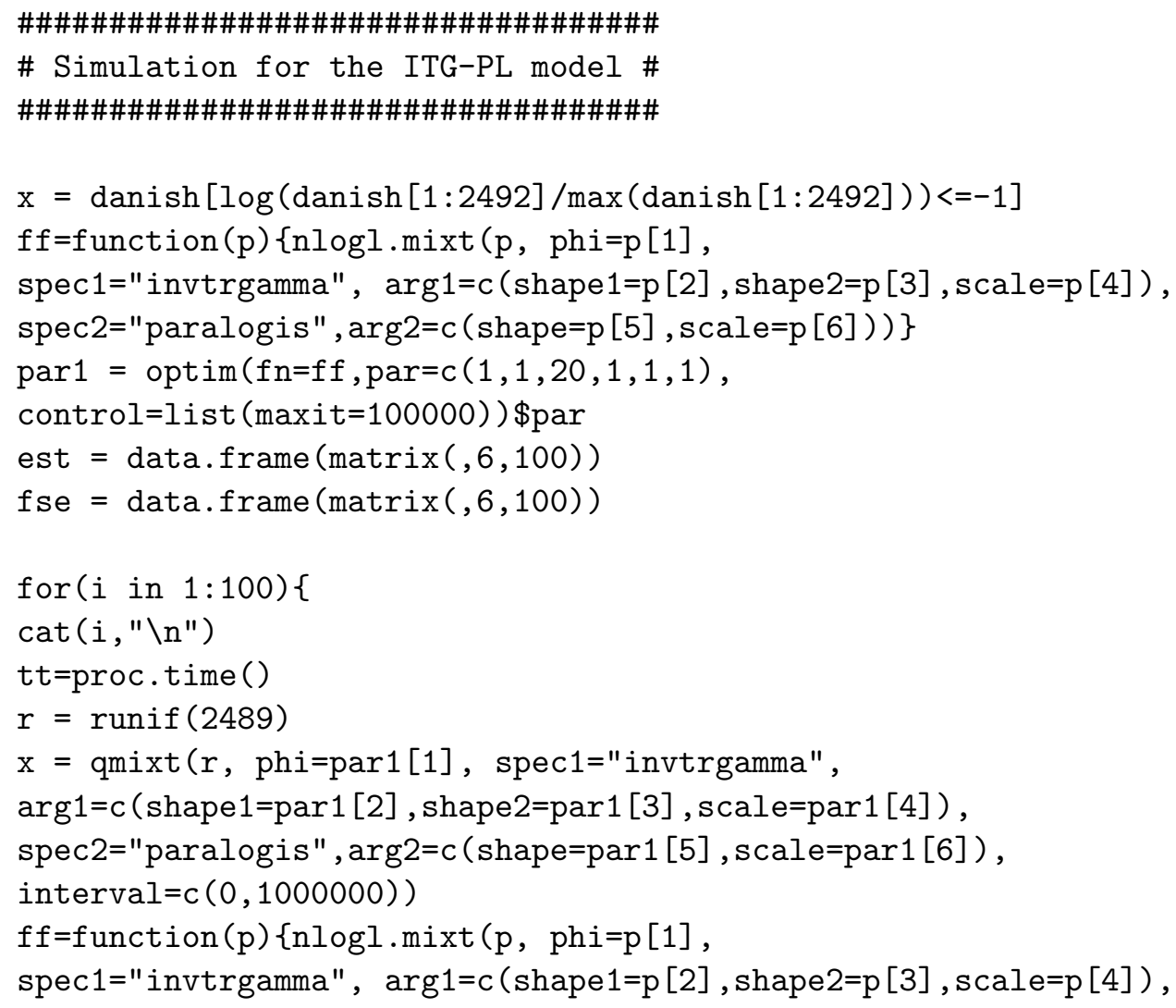




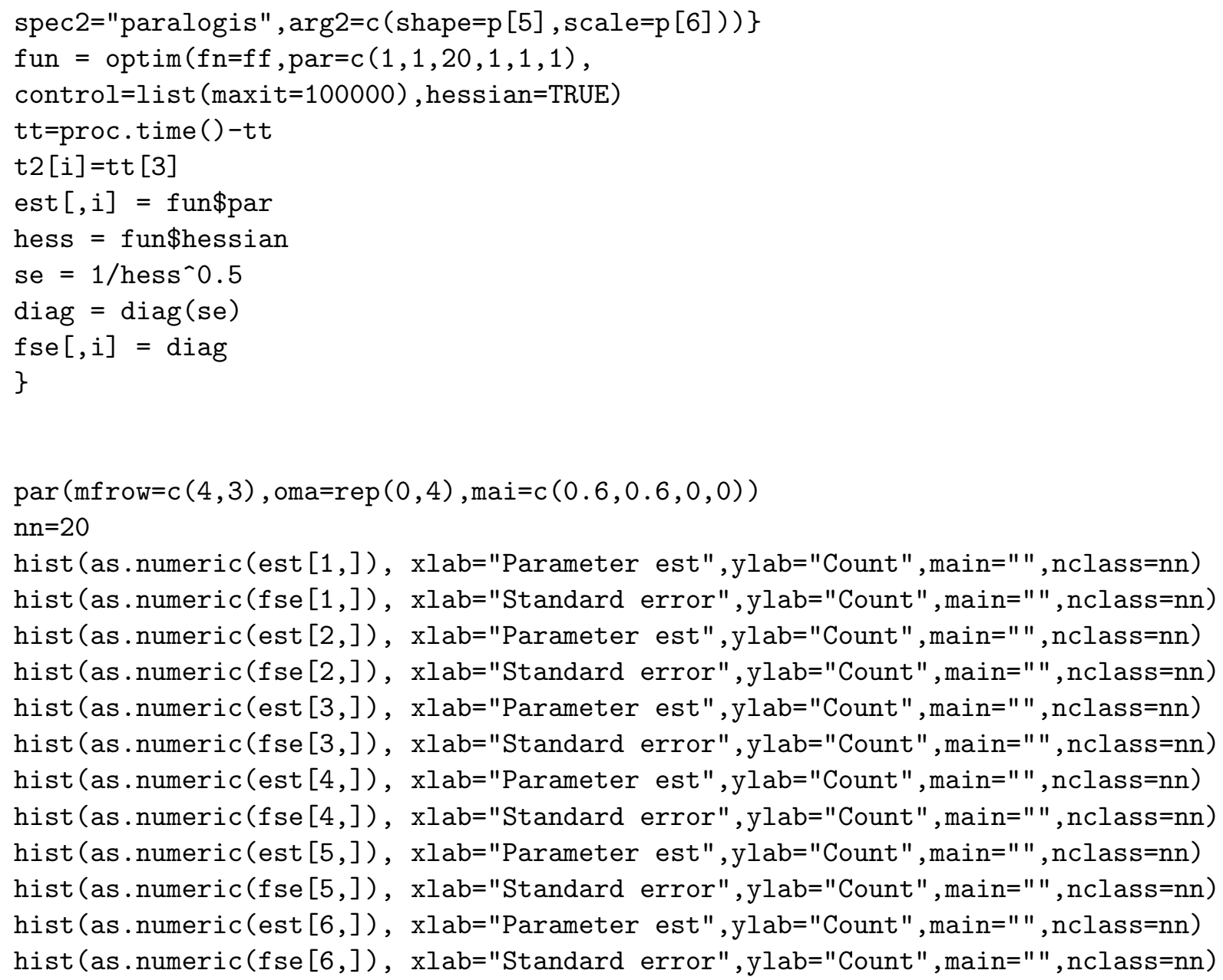




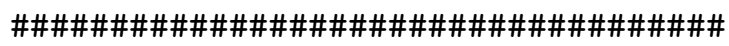

\# Simulation for the ITG-IPL model \#

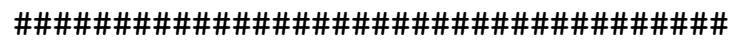

$x=\operatorname{danish}[\log (\operatorname{danish}[1: 2492] / \max (\operatorname{danish}[1: 2492]))<=-1]$

$f f=f u n c t i o n(p)\{n l o g l \cdot \operatorname{mixt}(p, p h i=p[1]$,

spec1="invtrgamma", $\arg 1=c($ shape1 $=p[2]$, shape2 $=p[3], s c a l e=p[4])$,

spec2="invparalogis", $\arg 2=c($ shape $=p[5]$, scale=p [6] )) $\}$

par1=optim $(f n=f f, \operatorname{par}=c(1,1,1,1,1,1)$,

control=list $(\operatorname{maxit}=100000))$ par

est $=$ data.frame $(\operatorname{matrix}(, 6,100))$

fse $=$ data.frame $(\operatorname{matrix}(, 6,100))$

for ( $i$ in $1: 100)\{$

cat ( $i, " \backslash n ")$

tt=proc.time ()

$r=\operatorname{runif}(2489)$

$\mathrm{x}=\operatorname{qmixt}(\mathrm{r}, \operatorname{ph} i=\operatorname{par} 1[1]$, spec1="invtrgamma",

$\arg 1=c($ shape1=par1 [2], shape2=par1 [3], scale=par1 [4] ),

spec2="invparalogis",

$\arg 2=c($ shape $=\operatorname{par} 1[5], \mathrm{scale}=\operatorname{par} 1[6])$, interval $=c(0,1000000))$

$f f=f$ unction $(p)\{$ nlogl $\operatorname{mixt}(p, p h i=p[1]$,

spec1="invtrgamma", $\arg 1=c($ shape1=p [2], shape2=p [3], scale=p [4] ),

spec2="invparalogis", $\arg 2=c($ shape $=p[5]$, scale=p [6] $))\}$

fun $=\operatorname{optim}(f n=f f, \operatorname{par}=c(1,1,1,1,1,1)$,

control=list (maxit $=100000)$, hessian=TRUE)

tt $=$ proc. time ()$-t t$

$\mathrm{t} 3[\mathrm{i}]=\mathrm{tt}[3]$

est $[, i]=$ fun $\$$ par

hess $=$ fun\$hessian

se $=1 /$ hess $^{\wedge} 0.5$

$\operatorname{diag}=\operatorname{diag}(\mathrm{se})$

fse $[, i]=$ diag

\}

$\operatorname{par}(\operatorname{mfrow}=\mathrm{c}(4,3)$, oma $=\mathrm{rep}(0,4), \operatorname{mai}=\mathrm{c}(0.6,0.6,0,0))$

$\mathrm{nn}=20$

hist (as.numeric(est $[1],), \mathrm{xlab}=$ "Parameter est",ylab="Count", main=" ",nclass=nn)

hist (as.numeric (fse $[1$,$] ), xlab="Standard error", ylab="Count", main=" ", nclass=nn)$

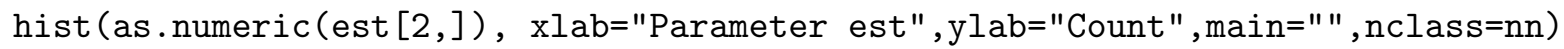

hist (as.numeric(fse [2,]), xlab="Standard error",ylab="Count", main=" ",nclass=nn)

hist (as.numeric (est $[3$,$] ), xlab="Parameter est", ylab="Count", main=" ", nclass=nn)$

hist (as.numeric (fse $[3$,$] ), xlab="Standard error", ylab="Count", main=" ", nclass=nn)$ 


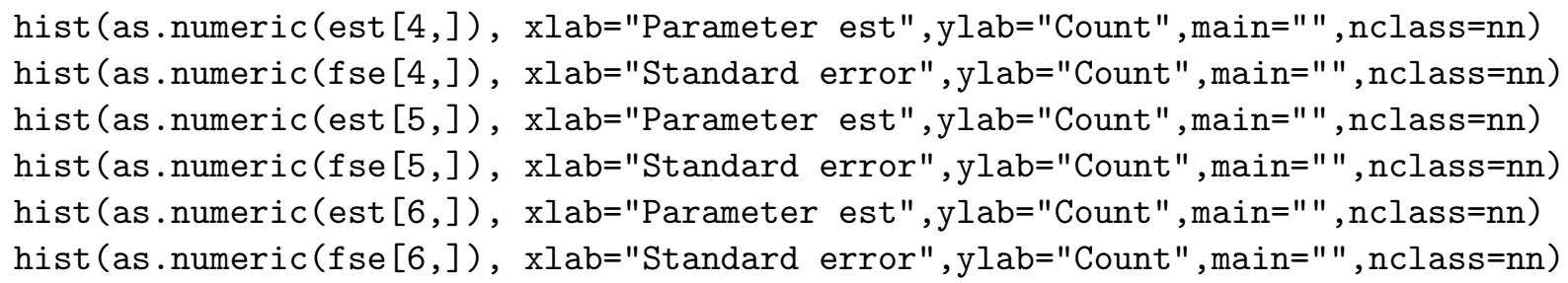

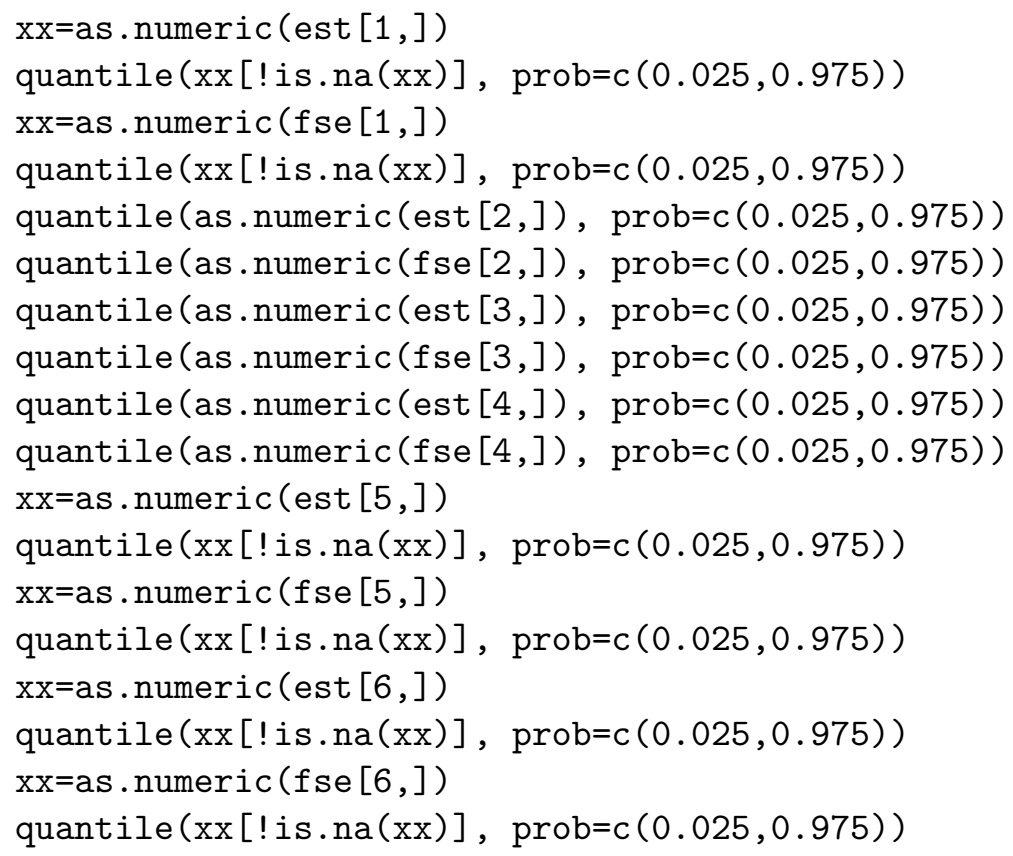

